\title{
Gravitational lens optical scalars in terms of energy-momentum distributions in the cosmological framework.
}

\author{
Ezequiel F. Boero, ${ }^{1 \star}$ Osvaldo M. Moreschi, ${ }^{2,3} \dagger$ \\ ${ }^{1}$ Instituto de Astronomía Teórica y Experimental (IATE), CONICET, Observatorio Astronómico de Córdoba, \\ Laprida 854, (X5000BGR) Córdoba, Argentina. \\ ${ }^{2}$ Facultad de Matemática, Astronomía, Física y Computación (FaMAF), Universidad Nacional de Córdoba, \\ Medina Allende S/N, X5000HUA, Córdoba, Argentina. \\ ${ }^{3}$ Instituto de Física Enrique Gaviola, IFEG, CONICET,Medina Allende S/N, X5000HUA, Córdoba, Argentina.
}

Accepted XXX. Received YYY; in original form ZZZ

\begin{abstract}
We present new results on gravitational lensing over cosmological RobertsonWalker backgrounds which extend and generalize previous works. Our expressions show the presence of new terms and factors which have been neglected in the literature on the subject. The new equations derived here for the optical scalars allow to deal with more general matter content including sources with non Newtonian components of the energy-momentum tensor and arbitrary motion. Our treatment is within the framework of weak gravitational lenses in which first order effects of the curvature are considered. We have been able to make all calculations without referring to the concept of deviation angle. This in turn, makes the presentation shorter but also allows for the consideration of global effects on the Robertson-Walker background that have been neglected in the literature.

We also discuss two intensity magnifications, that we define in this article; one coming from a natural geometrical construction in terms of the affine distance, that we here call $\tilde{\mu}$, and the other adapted to cosmological discussions in terms of the redshift, that we call $\mu^{\prime}$. We show that the natural intensity magnification $\tilde{\mu}$ coincides with the standard angular magnification $(\mu)$.
\end{abstract}

Key words: gravitational lensing: weak - gravitation - cosmology: observations

\section{INTRODUCTION}

This work generalizes the results of reference Gallo \& Moreschi (2011) to the cosmological framework in which a Robertson-Walker (R-W) geometry dominates the large scale structure of the spacetime. Therefore, we present new expressions for the lens optical scalars explicitly in terms of the local curvature associated to compact lenses in a cosmological scenario; and for the case of spherically symmetric lenses, we express the scalars directly in terms of the energy-momentum tensor distribution. We make no a priori assumptions on the nature of the lens, so that the new equations we present here admits a very general energy-matter content. In particular, they show the contribution from com-

\footnotetext{
* E-mail:boero@famaf.unc.edu.ar

† E-mail:o.moreschi@unc.edu.ar
}

ponents of the matter distribution that have been neglected previously.

The Universe is filled with objects and systems in motion, like black holes that have received a kick after a coalescence of a binary systemTichy \& Marronetti (2007); Baker et al. (2008); Civano et al. (2012); Gerosa \& Moore (2016), galaxies in a clusterLokas \& Mamon (2003), moving voidsLambas et al. (2016), etc. It is therefore important to have at hand a formalism that allows to study systems of gravitational lenses with motion. We therefore have made no assumption on the nature of the motion of the lenses and present new general expressions for the observable optical gravitational lens scalars, that improve on previous works on the subject of moving gravitational lensKopeikin \& Schaefer (1999); Frittelli (2003); Wucknitz \& Sperhake (2004).

We also present other new results including the relation between the intensity magnifications, that we define here, with the standard angular magnification. 
In our previous workGallo \& Moreschi (2011) on gravitational lenses, we have presented new general expressions for the bending angle and the optical scalars for a congruence of null geodesics in the regime of weak gravitational lensing over a flat background. These formulas have the advantage of being explicitly gauge invariant and allow us to include more general forms of matter distributions than those discussed in most standard references on the subjectSchneider et al. (1992); Seitz et al. (1994); Wambsganss (1998); Bartelmann (2010).

Systems involving gravitational lens effects appear naturally in the cosmological context. And although the standard works on gravitational lens, based on a flat background, are useful; new subtle issues arise when the gravitational lens is studied in the cosmological framework. We improve on the tools shown in Gallo \& Moreschi (2011), and so provide with new expressions, for weak gravitational lenses, that are useful for the study of the matter content in cosmological astrophysical systems. Indeed, the expressions presented below are a tool to study in more detail the missing mass problem; since we present equations describing gravitational lensing that describe more general situations; enabling the characterization of more general energy momentum tensors, in contrast to the usual Newtonian description of the dark matter phenomena. Our expressions do not neglect spacelike components of the energy momentum tensor. In fact, we have seen in the past that there is some suggestive evidence that the inclusion of the up to now neglected spacelike components of the energy momentum tensor turns out to be important for the study of dark matter phenomena as it was shown in Gallo \& Moreschi (2012) where a simplified model with this features allows for excellent description of the matter content in clusters of galaxies, and for the velocity rotation curves in galaxies.

It is probably worthwhile to mention that all the astrophysical observations of the gravitational lens optical scalars, namely of the expansion and the shear, give results whose values are much less than the unit value. For this reason one is convinced that pursuing calculations of weak gravitational lenses taking into account the linear effects of the curvature is enough to explain the observational data. In any case our starting point will be the exact geodesic deviation equation; which happened to be linear in the curvature. To be more precise, below we will present a decomposition of the curvature in terms of a background term, that we will call $Q_{B}$, and an extra term, that will call $Q_{L}$. By weak gravitational lens effects we mean those that are deduced from the geodesic deviation equation at first order in the extra terms of the curvature fields: namely, $Q_{L}$. In this decomposition of the total curvature in terms of the background plus a lens term, it is not assumed that the additional term must be small in any sense. For example, $Q_{L}$ could be the fields that represent locally a Schwarzschild black hole, or a $90 \%$ deficit in the cosmological density in the case of a void. That is we are not assuming at this stage any kind of perturbation; it is just the representation of an exact geometry, without perturbation, in terms of a decomposition with respect to a chosen background. The details and implications of this statement will become clear along the article. However we advance that the effects of the background curvature will be calculated exactly; so that our final equations will have quadratic terms of order $\mathscr{O}\left(Q_{B} Q_{L}\right)$.
In this work, our main focus is to broaden the range of validity of previous results and discussions to the case in which the background is within the family of RobertsonWalker geometries, proceeding in a systematic way without introducing early assumptions of Newtonian character and further preconditions related to the observational configuration such as the well known approximation of thin lenses. Regarding this last point, here, instead we have chosen to consider such approximations after a general treatment of the exact equations governing the distortion of the images in the cosmological background. The new improvements that we introduce require subtle but significant modifications in the treatment of the subject, that will be described along the article. In particular, whenever appropriate we will mark the difference with the simplistic approach to the subject in which all the physics of gravitational lenses is encoded in the quotient of the projected surface mass density by the so called critical mass density, normally denoted by $\Sigma / \Sigma_{c r}$ Schneider et al. $(1992,2006)$. An interesting new result is for example the presence of a redshift factor correcting the widely used expressions of thin lenses as appears in eq. (51), part 1 of reference Schneider et al. (2006) or eq. (16) of reference Wambsganss (1998) (See section 9). Our findings are relevant to works concerning tests of fundamental geometrical relations in observational cosmology which are based on the use of gravitational lensing; see for example Holanda et al. (2016); Liao et al. (2016).

Let us mention here briefly other differences of our approach to the subject of cosmic lenses with the standard references. It has been suggested and is also widely believed that when describing the gravitational lens optical scalars in the cosmological framework one only needs to replace, in the flat background discussions, any appearance of distance with the area distance, or angular diameter distanceSchneider et al. (2006); we point out below that this imposes severe limitation on the class of systems one can treat with this technique, and provide with the general equations. In other occasions the presentation of gravitational lens physics relays on the concept of effective local index of refractionBartelmann \& Schneider (2001) which can only be defined in very especial casesSchneider et al. (1992). In contrast, our approach is to deduced the most general gravitational lens equations that can be used in the cosmological framework. In the process of obtaining these equations it is essential to review the main concepts involved in the problem of gravitational lens so that gauge invariant quantities can be derived without the use of initial simplifying assumptions. For this reason we spend some time below to make this review; which allows us for example to claim that the only sensible notion of distance that one should use when referring to observables in problems involving gravitational lenses is the affine distance as defined by the observer. This does not contradict the use of area distance in calculations, rather it is in contrast to the claim that one should only use the angular diameter distance in gravitational lens discussions in the cosmic framework. We therefore emphasize along the article that the derived concepts coming from gravitational lens effects concern always to comparisons referring to same affine distances. This approach lead us to define the concept of intensity magnification that to our knowledge has not been used in the past.

In section 2 we spent some time reviewing the basic con- 
cepts, also with the intention to set the notation and language used in the rest of this paper. In section 3 we discuss null geodesics in R-W geometry and coordinates adapted to the observations. Section 4 contains a discussion of the different notions of distance appearing in cosmological studies together with the relation among them. We discuss different notions of magnifications in section 5 . Next, in section 6 we present the expression for the cosmological convergence, valid for arbitrary angular aperture and without approximations, when the R-W geometry is considered as a gravitational lens. The appearance of an additional lens over the $\mathrm{R}$-W background is discussed in section 7; where our generalized expression are presented. In this section the presentation deals with compact lenses of small extension in comparison with the cosmological dimension traversed by the photons; and the calculations of the optical scalars are carried out within the framework of weak gravitational lenses; in which first order effects of the curvature are used. Section 8 deals with the axially symmetric case. In section 9 we present the usual approximation of thin lenses; while in section 10 the equations for a static spherically symmetric lens are written in its most general form. Final comments are included in section 11. Lastly, in the appendix we include the geometrical structure of the cosmological background in terms of the less common presentation associated to a null tetrad which is widely used in our approach.

\section{GRAVITATIONAL LENSES}

\subsection{Preliminaries}

The first thing to do is to agree in the language one would use to describe the phenomenology of gravitational lens effects. The basic idea one has in mind is depicted in figure 1; where it is sketched the situation of an observer, at the bottom of the figure, receiving light from a source at the furthest surface, which is affected by a gravitational lens, along the path of the rays.

In the discussion of the gravitational lens effects coming from an homogeneous cosmological spacetime, one would not have a lens at a particular distance, since the whole spacetime acts as a lens; however we will retain the basic notions for the observed angle $\theta$ and would observe angle $\beta$; where the word 'would' refers to what the astrophysicist would expect, if there where no gravitational lens effect, and therefore no curvature.

In any case, one can use the standard definitions of convergence and shear, that comes from the relation of these angles.

Given a related pair of directions, represented by angles $(\beta, \theta)$, one can consider a small variation from them, whose difference will be related by a linear relation. In the sphere of directions, they can be expressed as:

$\delta \beta^{a}=\mathcal{A}_{b}^{a} \delta \theta^{b}$,

where the matrix $\mathcal{A}_{b}^{a}$ is in turn expressed by

$\mathcal{A}_{b}^{a}=\left(\begin{array}{cc}1-\kappa-\gamma_{1} & -\gamma_{2} \\ -\gamma_{2} & 1-\kappa+\gamma_{1}\end{array}\right)$;

where the optical scalars $\kappa, \gamma_{1}$ and $\gamma_{2}$, are known as convergence $\kappa$ and shear components $\left\{\gamma_{1}, \gamma_{2}\right\}$, and have the information of distortion of the image of the source due to

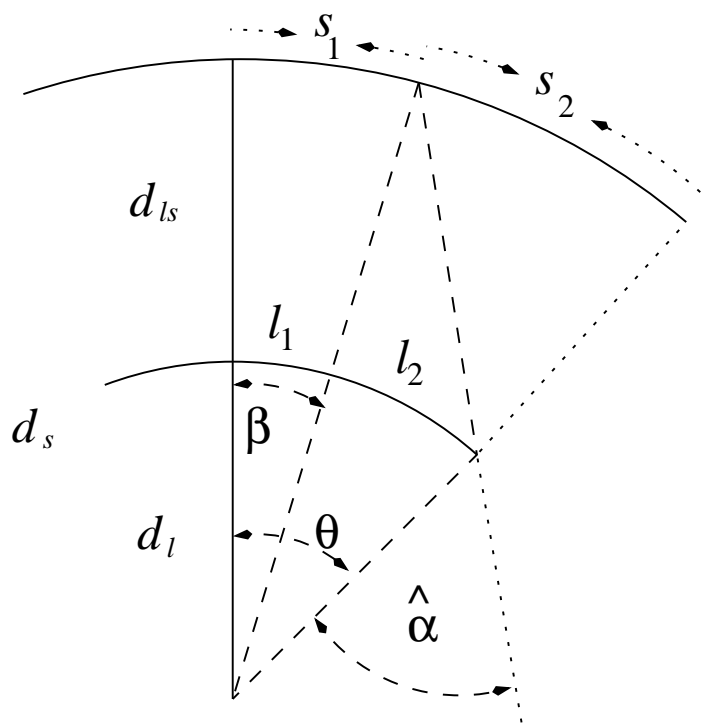

Figure 1. This graph shows the basic and familiar angular variables in terms of a simple flat background geometry. The letter $s$ denote sources, the letter $l$ denotes lens and the observer is assumed to be situated at the apex of the rays.

the lens effects. In fact the shear is normally represented by the complex quantity $\gamma_{c}=\gamma_{1}+i \gamma_{2}$.

It should be emphasized that since the definition of $\delta \beta^{a}$ and $\delta \theta^{b}$ refer to two different geometries, one must say at what distance is the source in both cases. The calculations of the gravitational lens effects are carried out using as fundamental variable the affine distance $\lambda$ from the point of observationGallo \& Moreschi (2011), and with respect to the four velocity of the observer. This point is also related to the notion of angular diameter distance, that we will discuss again below for the case of homogeneous spacetimes; but here we will only use the notion that a rod of length $\delta l$ which is observed to subtend an angle $\delta \phi$ defines the angular diameter distance $D_{A}$ by:

$\delta l=D_{A} \delta \phi$.

For each spacetime one has one notion of observed angular diameter distance. In the above equation, one assumes the observation of some object of size $\delta l$, so that one would have

$\delta l=D_{A}(\eta) \delta \beta$

and

$\delta l=D_{A}(g) \delta \theta ;$

where $\eta$ is the flat metric and $g$ the real physical metric. In Minkowski spacetime the angular diameter distance coincides with the affine distance, namely: $D_{A}(\eta)=\lambda$. In a general spacetime, with metric $g$, one has the source at the affine distance $\lambda_{s}$; therefore, in order to make the comparison in equation (1) one takes $\lambda_{s}=\lambda$.

\subsection{The null geodesic congruence}

Here we provide with a brief summary of the essentials equations needed to the treatment of gravitational lensing. 
Let $u$ be an scalar function such that the level sets $u=$ constant denote a family of null hypersurfaces; then we define the vector field $\ell^{a}$ from the one-form

$\ell_{a}=\nabla_{a} u=(d u)_{a} ;$

which satisfies

$\ell^{a} \ell_{a}=0$,

and

$\ell^{a} \nabla_{a} \ell^{b}=0$.

Therefore, there is a natural radial affine function $r$, given by

$\ell^{a}=\left(\frac{\partial}{\partial r}\right)^{a}$.

Let us note that by construction this congruence is twist free.

In our construction $u=$ constant will characterize our past null cone.

The way in which the null function $u$ grows to the future is normalized by the condition

$v^{a} \ell_{a}=1$;

where $v^{a}$ is the observer's 4 -velocity. This condition implies that we are choosing $\ell^{a}$ to be future pointing; and it also fixes the scale of the affine parametrization, but it still remains a freedom associated to the choice of origin; or equivalently the freedom to make a translation in the value of $r$, for each null direction in the congruence. We will fix below this freedom by taking the natural choice which correspond to set $r=0$ at the apex of the cone.

In what follows it will be useful to refer the discussion to a null tetrad adapted to the past null cone of the observer. The first null vector in our tetrad will be $\ell^{a}$ in the way we have just defined. The other vectors are two spacelike vector fields $m^{a}$ and $\bar{m}^{a}$ tangents to the surfaces $r=$ constant and $u=$ constant, and satisfying

$m^{a} \bar{m}_{a}=-1$

and the last null vector will be denoted as $n^{a}$ which is chosen orthogonal to $m^{a}$ and $\bar{m}^{a}$ and satisfying

$n^{a} \ell_{a}=1$.

\subsection{The geodesic deviation equation}

The main object for the quantification of the optical distortions is the deviation vector $\varsigma^{a}$ which describes the behaviour of the congruence by computing the local separation of different geodesics. By definition it satisfies the condition

$\mathscr{L}_{\ell} \varsigma^{a}=0$

that is, the Lie derivative along $\ell^{a}$ vanishes.

The equation that provides us with the information about the distortion of images is the geodesic deviation equation for the deviation vector $\varsigma^{a}$ along the congruence with tangent $\ell^{a}$; namely

$\ell^{a} \nabla_{a}\left(\ell^{b} \nabla_{b} \varsigma^{d}\right)=R_{a b c}{ }^{d} \ell^{a} \varsigma^{b} \ell^{c}$,

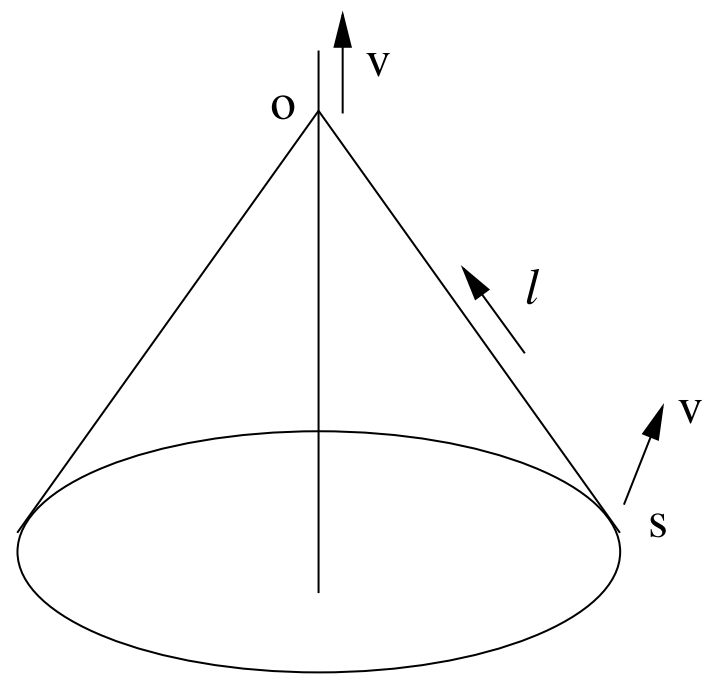

Figure 2. This graph shows schematically a spacetime diagram with the location of the observer "o", with its velocity, a source "s", with its velocity, and the trajectory of a photon with null vector $\ell$.

where $R_{a b c}{ }^{d}$ denotes the Riemann tensor. This equation is obtained by taking a second covariant derivative to the deviation vector.

The deviation vector can be expressed as

$\varsigma^{a}=\varsigma \bar{m}^{a}+\bar{\varsigma} m^{a} ;$

and using the vector $\mathcal{X}$ build up from the components $\{\varsigma, \bar{\varsigma}\}$;

$\mathcal{X}=\left(\begin{array}{l}\varsigma \\ \bar{\varsigma}\end{array}\right)$

equation (13) can be written in the following form as

$\ell(\mathcal{X})=-P \mathcal{X}$

where the matrix $P$ is

$P=\left(\begin{array}{cc}\rho & \sigma \\ \bar{\sigma} & \bar{\rho}\end{array}\right)$,

and $\rho, \sigma$ are the spin coefficients in the GHPGeroch et al. (1973) notation which correspond respectively to the expansion and the shear of the congruence under study. We would like to notice that the symbol for the spin coefficient $\rho$ should not be confused with the symbol $\varrho$ that we will later use for denoting energy density.

It can be seen that the matrix $P$ satisfies

$\ell(P)=P^{2}+Q$;

where $Q$ is the matrix

$Q=\left(\begin{array}{cc}\Phi_{00} & \Psi_{0} \\ \bar{\Psi}_{0} & \Phi_{00}\end{array}\right)$

whose elements are the curvature components:

$\Phi_{00}=-\frac{1}{2} R_{a b} \ell^{a} \ell^{b}$

and

$\Psi_{0}=C_{a b c d} \ell^{a} m^{b} \ell^{c} m^{d} ;$

denoting with $R_{a b}$ the Ricci tensor and $C_{a b c d}$ the Weyl tensor. The equations that describe the behavior of, what we 
now call the spin coefficients, have been discussed extensively in the literature, including the seminal works of R.K. SachsSachs (1961).

The second order differential equation coming from the geodesic deviation equation is; using this notation:

$\ell(\ell(\mathcal{X}))=-Q \mathcal{X}$.

Working with this equation has the advantage that it is explicitly given in terms of the curvature components.

\section{NULL GEODESICS IN ROBERTSON-WALKER SPACETIMES}

As it is well known R-W metrics are geometries which posses spatially homogeneous and isotropic symmetryWald (1984) and play a fundamental role in our standard description of the Universe at its largest scales.

The line element of such geometries can be cast in the following form

$d s^{2}=d t^{2}-A^{2}(t) d L_{k}^{2} ;$

where $t$ is the so-called cosmological time, i.e. the proper time associated to the family of fundamental observers which perceives isotropy and $d L_{k}^{2}$ is the line element for the spacelike surfaces orthogonal to the preferred observers. As a consequence of the symmetries $d L_{k}^{2}$ must to be one of the three possible 3-Riemaniann metrics of constant curvature. We distinguish them by the subindex $k$ which takes the values $k=1, k=0$ and $k=-1$ corresponding respectively to a 3 -sphere, a 3 -plane and a 3 -hyperboloid. Three equivalent and useful ways of write $d L_{k}^{2}$ are:

$d L_{k}^{2}=d \chi^{2}+f_{k}^{2}(\chi) d \Sigma^{2}$,

$d L_{k}^{2}=\frac{d r^{2}}{1-k r^{2}}+r^{2} d \Sigma^{2}$,

$d L_{k}^{2}=\frac{1}{\left(1+\frac{k}{4} r^{2}\right)^{2}}\left(d r^{2}+r^{2} d \Sigma^{2}\right) ;$

where in equation $(25) f_{k}(\chi)$ is given by

$f_{k}(\chi)= \begin{cases}\sinh (\chi), & \text { for } k=-1, \quad 0 \leqslant \chi<\infty, \\ \chi, & \text { for } k=0, \quad 0 \leqslant \chi<\infty \\ \sin (\chi), & \text { for } k=1, \quad 0 \leqslant \chi \leqslant \pi .\end{cases}$

The two-dimensional line element $d \Sigma^{2}$ is the common metric of the unit 2-sphere, which is often found in one of the two following forms

$d \Sigma^{2}=d \theta^{2}+\sin ^{2}(\theta) d \phi^{2}$,

$d \Sigma^{2}=\frac{4}{(1+\zeta \bar{\zeta})^{2}} d \zeta d \bar{\zeta} \equiv \frac{1}{P_{0}^{2}} d \zeta d \bar{\zeta} ;$

where $(\theta, \phi)$ are usual angular coordinates and $(\zeta, \bar{\zeta})$ complex stereographic coordinates on the complex plane.

\subsection{Past light cone of preferred observers and associated null tetrads}

Regarding to the information inferred from observations in our past light cone one realizes that the relevant geometrical quantities for the description of observations are the null rays reaching the observer and the angular deviations of such rays. All observable quantities are constructed from them. Then, it is necessary to have at hand an adequate description in terms of the past light cone of the observers.

Associated to the observer one has its proper time $\tau$; which for co-moving observers coincides with the standard coordinate time $t$. Then, the $4-$ velocity $v^{a}$ of the observer, can be expressed as

$v^{a}=\left(\frac{\partial}{\partial t}\right)^{a}$.

In the following we will introduce a coordinate system which is well adapted to the discussion that follows. We begin by labeling each past light cone with a null function $u$ which takes constant values on the cones. In particular, this means that the value of $u$ can be set by the proper time $\tau$; an we choose the relation

$\frac{d u}{d \tau}=1 ;$

at the origin; but one can also use another specification for $u$ that is not based on the synchronization of the null function with the proper time of the observer. In fact, we will use below a definition based on the conformal structure of the metric. But in any case, the physically relevant null geodesic congruence associated to the observer, can be expressed in terms of this null function by (6).

Let us note also that this setup can be generalized for an arbitrary world line $\gamma_{0}^{\prime}$ with $4-$ velocity $v^{\prime a}$ in terms of a general proper time $\tau^{\prime}$.

\subsubsection{The conformal advanced null function $\tilde{u}$}

Let us introduce another null function which is very useful for the calculations. We define $\tilde{u}$ from

$d \tilde{u}=\frac{d t}{A(t)}+d \chi$

where we have used tilde in order to differentiate it from the natural choice presented before.

This choice is appropriated to the study of the asymptotic behaviour of the geometry Moreschi (1990) but it is also helpful in many computation in comparison with the choice $u=\tau$.

The next step is to complete the coordinate system putting coordinates on each past light cone. In this process also a null tetrad $\left(\tilde{\ell}^{a}, \tilde{m}^{a}, \tilde{\bar{m}}^{a}, \tilde{n}^{a}\right)$ associated to this construction can be selected.

First let us consider the vector $\tilde{\ell}^{a}$ determined by the one-form

$\tilde{\ell}=d \tilde{u}$

which is geodesic and affinely parametrized, since $\tilde{u}$ is null.

Now, let $\tilde{r}$ be the affine parameter along the cone associated to $\tilde{\ell}^{a}$, starting from the observer. This will be our second coordinate. Then, a pair of null complex vectors $\tilde{m}^{a}$ and $\tilde{\bar{m}}^{a}$ can be chosen to be tangent to the surfaces $\tilde{u}=$ constant and $\tilde{r}=$ constant and satisfying

$\tilde{m}^{a} \tilde{\bar{m}}_{a}=-1$.

To complete our coordinate system we label the generators of the null cone by the stereographic coordinates $(\zeta, \bar{\zeta})$. 
Finally, we also complete our tetrad with a null vector $\tilde{n}^{a}$ chosen such that

$$
\begin{aligned}
\tilde{n}^{a} \tilde{m}_{a} & =0, \\
\tilde{n}^{a} \tilde{\ell}_{a} & =1 .
\end{aligned}
$$

Explicitly, in term of the usual coordinates $(t, \chi, \zeta, \bar{\zeta})$, the vector field $\tilde{\ell}^{a}$ is expressed as:

$\tilde{\ell}=\frac{1}{A(t)}\left(\frac{\partial}{\partial t}\right)-\frac{1}{A^{2}(t)}\left(\frac{\partial}{\partial \chi}\right) ;$

while for the other tetrad vectors we have:

$$
\begin{aligned}
\tilde{m} & =\frac{\sqrt{2} P_{0}}{A(t) f_{k}(\chi)}\left(\frac{\partial}{\partial \zeta}\right), \\
\tilde{m} & =\frac{\sqrt{2} P_{0}}{A(t) f_{k}(\chi)}\left(\frac{\partial}{\partial \bar{\zeta}}\right), \\
\tilde{n} & =\frac{A(t)}{2}\left[\left(\frac{\partial}{\partial t}\right)+\frac{1}{A(t)}\left(\frac{\partial}{\partial \chi}\right)\right] .
\end{aligned}
$$

The line element (24) with the choice (25) can be expressed in terms of the new coordinate system $(\tilde{u}, \tilde{r}, \zeta, \bar{\zeta})$.

We will do this with an intermediate step; using eq. (33) one can express the geometry in terms of the coordinate system $(\tilde{u}, \chi, \zeta, \bar{\zeta})$ to find:

$d s^{2}=A^{2}(t)\left(d \tilde{u}^{2}-2 d \tilde{u} d \chi-f_{k}^{2} d \Sigma^{2}\right) ;$

where here it is important to note that the vector $\tilde{\ell}$ is expressed as

$\tilde{\ell}=-\frac{1}{A^{2}(t(\tilde{u}, \chi))}\left(\frac{\partial}{\partial \chi}\right)$

Since also $\tilde{\ell}=\frac{\partial}{\partial \tilde{r}}$, we also have the relation

$$
\frac{\partial \chi}{\partial \tilde{r}}=-\frac{1}{A^{2}(t(\tilde{u}, \chi))} ;
$$

so we take

$\tilde{r}=-\int_{0}^{\chi} A^{2}\left(t\left(\tilde{u}, \chi^{\prime}\right)\right) d \chi^{\prime}=\int_{t(\tilde{u}, \chi=0)}^{t(\tilde{u}, \chi)} A\left(t^{\prime}\right) d t^{\prime}$.

This is the relation that we need to cast the line element in terms of the coordinates $(\tilde{u}, \tilde{r}, \zeta, \bar{\zeta})$ since now we can differentiate to obtain

$d \tilde{r}=\left(A^{2}(t(\tilde{u}, \chi))-A^{2}\left(t_{0}\right)\right) d \tilde{u}-A^{2}(t(\tilde{u}, \chi)) d \chi ;$

where we use the notation $A\left(t_{0}\right)$ to mean

$A\left(t_{0}\right) \equiv A(t(\tilde{u}, \chi=0)) ;$

where it should be noted that $t_{0}=t(\tilde{u}, \chi=0)$ need not be the present time; but the coordinate $t$ evaluated at the origin.

Then, one arrives to the desired expression for the metric:

$d s^{2}=\left(2 A^{2}\left(t_{0}\right)-A^{2}(t)\right) d \tilde{u}^{2}+2 d \tilde{u} d \tilde{r}-A^{2}(t) f_{k}^{2}(\chi) d \Sigma^{2}$.

For completeness we include here the relations among the differential of the old coordinate system in terms of the latest one; they are given as follows:

$d t=\frac{A^{2}\left(t_{0}\right)}{A(t)} d \tilde{u}+\frac{1}{A(t)} d \tilde{r}$,

$d \chi=\left(1-\frac{A^{2}\left(t_{0}\right)}{A^{2}(t)}\right) d \tilde{u}-\frac{1}{A^{2}(t)} d \tilde{r}$.

\subsubsection{The inertial advanced null function $u$}

In this case we consider the choice $u=\tau$ which will be presented in similar way to the discussion of the previous section. This has the advantage to help the reading and at the same time it allows to arrive to the main expression in a simple way using the definitions presented above.

Then, let us find the relation between both functions by noting that

$v(\tilde{u})=\frac{d \tilde{u}}{d u}$

along the world line of a preferred observer. Since $u$ is normalized with $t$ at the origin, from equation (33) one has

$d u=A\left(t_{0}\right) d \tilde{u}=A(t(\tilde{u}, \chi=0)) d \tilde{u} ;$

which leads us to define the first null vector $\ell$ as

$\ell=d u$.

This gives us a congruence of future directed null geodesics, affinely parametrized that reach the observer. The affine parameter in this case will be denoted by $r$ and constitutes our second coordinate. Stereographic coordinates label the generators of the congruence as before.

The null vector $\ell$ can be expressed in the following way:

$\ell=\frac{A\left(t_{0}\right)}{A(t)}\left[\left(\frac{\partial}{\partial t}\right)-\frac{1}{A(t)}\left(\frac{\partial}{\partial \chi}\right)\right]$

while the other vectors of the tetrad can be taken to be

$m=\frac{\sqrt{2} P_{0}}{A(t) f_{k}(\chi)}\left(\frac{\partial}{\partial \zeta}\right)$

$\bar{m}=\frac{\sqrt{2} P_{0}}{A(t) f_{k}(\chi)}\left(\frac{\partial}{\partial \bar{\zeta}}\right)$,

$n=\frac{1}{2} \frac{A(t)}{A\left(t_{0}\right)}\left[\left(\frac{\partial}{\partial t}\right)+\frac{1}{A(t)}\left(\frac{\partial}{\partial \chi}\right)\right]$.

We left for the appendix A a discussion with the details of the connection and the curvature of the geometry using the null tetrad introduced above.

The metric in the advanced coordinates $(u, \chi, \zeta, \bar{\zeta})$ can be expressed as

$d s^{2}=A^{2}(t)\left(\frac{d u^{2}}{A^{2}\left(t_{0}\right)}-2 \frac{d u d \chi}{A\left(t_{0}\right)}-f_{k}^{2}(\chi) d \Sigma^{2}\right)$.

The affine parameter $r$ associated to $\ell$ and the differential $d r$ are found to be

$r(u, \chi)=\frac{1}{A\left(t_{0}\right)} \int_{t(u, \chi=0)}^{t(u, \chi)} A\left(t^{\prime}\right) d t^{\prime}$,

and

$d r=-\frac{A^{2}(u, \chi)}{A\left(t_{0}\right)} d \chi-\left(1-\frac{A^{2}(u, \chi)}{A^{2}\left(t_{0}\right)}+r H\left(t_{0}\right)\right) d u ;$

where we introduce here the Hubble function on the observer world line

$H\left(t_{0}\right) \equiv \frac{1}{A\left(t_{0}\right)} \frac{d A(t(u, \chi=0))}{d u}$.

One can then transform to a coordinate system in which the radial coordinate is $r$ instead of $\chi$; so that now $t=t(u, r)$ 
and $\chi(u, r)$. Note that now $t_{0}=t(u, r=0)$. Gathering together this expressions one arrives to the metric in coordinates $(u, r, \zeta, \bar{\zeta})$ :

$$
\begin{aligned}
d s^{2}= & 2\left(1-\frac{A^{2}(t)}{2 A^{2}\left(t_{0}\right)}+r H\left(t_{0}\right)\right) d u^{2}+2 d u d r \\
& -A^{2}(t) f_{k}^{2}(\chi) d \Sigma^{2} .
\end{aligned}
$$

\subsection{Deviation vector for a radial congruence of null geodesics}

In the $\mathrm{R}-\mathrm{W}$ geometries, the behaviour of the deviation vector, is easily found by considering the ordinary linear differential equation (13) on the past null cone:

$\ell(\mathcal{X})=-\rho \mathcal{X}$.

Let us note here that we can use $\ell^{a}$ or $\tilde{\ell}^{a}$ as vector field tangent to the congruence since the description is independent of the affine parametrization.

In the coordinates $(u, r)$ of section 3.1.2 the convergence $\rho$ takes the simple and transparent expression:

$\rho=-\frac{1}{A(t) f_{k}(\chi)} \ell\left(A(t) f_{k}(\chi)\right)$.

This means that the deviation vector is given by:

$\mathcal{X}=A(t) f_{k}(\chi) \mathcal{V}_{0}$

where the integration constant $\mathcal{V}_{0}$ has the meaning of the angle measured by the fundamental observer between two neighbour null geodesic.

Since this factor will appear frequently we define

$D_{A}(t, \chi) \equiv A(t) f_{k}(\chi) ;$

which will be identified below to the angular diameter distance.

\section{DIFFERENT NOTIONS OF DISTANCE}

Since the notion of distance plays a central role in the discussion of gravitational lenses, and it is fundamental to all the discussions in this paper, it is convenient that we review here the usual notions of distance that appear in the cosmological framework.

\subsection{The redshift}

The observer $o$ defines the redshift associated with the event $s$ by the expression

$1+z=\frac{\left(l^{a} v_{a}\right)_{s}}{\left(l^{a} v_{a}\right)_{o}}$

where $l^{a}$ is the tangent vector to a null geodesic connecting the two events and $v^{a}$ denotes either the 4 -velocities of the source $s$ or that of the observer.

Although the redshift is an indication of different proper times measures it is also used as an indication of distance in R-W spacetimes. When the source and the observer are both fundamental observers of a R-W spacetime, one has

$1+z=\frac{A\left(t_{0}\right)}{A(t)}$.
In other words, the redshift can be used as a notion of distance if one has at hand a given family of cosmological objects with known motion.

\subsection{Geometric distance or normalized affine distance}

Given an observer with 4 -velocity $v^{a}$, there is a natural notion of distance $\lambda$ defined on any past directed null geodesic on any spacetime; which is identified with the affine parameter so chosen that normalization (10) holds, and is zero at the observer position. In the case of a $\mathrm{R}-\mathrm{W}$ spacetime, this distance is related to the coordinate and affine parameter $r$, by

$\lambda=-r$.

To differentiate from other definitions we will call this the geometric distance or normalized affine distance. By definition it increases monotonically along every past directed null geodesic from the observer.

A fundamental concept in the study of gravitational lenses is the comparison of an image that is observed, with the unaffected image, for which gravitational effects can be completely neglected. But, then, it is crucial to compare the images in both situations when the source is at the 'same' distance. It is here that the definition of the distance that one should use, becomes important, and we claim that the appropriate notion is the one given by the definition of normalized affine distance, or geometric distance. This is the concept that appears as the dynamical parameter in the fundamental equation (23), that is always well behaved (it increases monotonically as the objects are further away) and it can be applied to any situation and spacetime (with or without symmetries), and that we use as the fundamental notion of distance in this work.

\subsection{Angular diameter distance and future angular distance}

\subsubsection{Angular diameter distance and area distance}

The so called 'angular diameter distance', $D_{A}$, is defined as

$d l=D_{A} d \theta$

where $d l$ is the projected size of the object, perpendicular to the line of sight, at the place of the emitter, and $d \theta$ is the subtended angle measured in the sky by the observer.

This definition is closely related to the area distance; which is defined from:

$d A=\left|D_{+} D_{-}\right| d \Omega ;$

where $d A$ is the projected area of the object, perpendicular to the line of sight, at the place of the emitter, $D_{+}$and $D_{-}$are the angular diameter distances along the principal directions of the areaPerlick (2004), and $d \Omega$ is the solid angle seeing by the observer; and the area distance $D_{\text {area }}$ is defined by

$D_{\text {area }}=\sqrt{\left|D_{+} D_{-}\right|}$

so that now one can write

$d A=D_{\text {area }}^{2} d \Omega$. 
Let us note that this measure of distance does not depend on the peculiar velocity of the emitter, it depends only on the 4-velocity of the observerPerlick (2004).

It is probably important to emphasize that in the discussion on R-W spacetimes the area distance coincides with the angular diameter distance; for this reason in the remainder of the paper will just use $D_{A}\left(=D_{\text {area }}\right)$ to denote both distances, in the case of $\mathrm{R}-\mathrm{W}$ spacetimes.

For fundamental observers in R-W geometries this notion of distance is explicitly given by those presented in equation (66):

$D_{A}=A(t(u, r)) f_{k}(\chi(u, r))$.

From its definition, one notes that the area distance is related to the expansion of a thin bundle of null geodesics leaving the observer and reaching the source; in fact, in a $\mathrm{R}-\mathrm{W}$ spacetime one can see this fact explicitly by looking at equation (64). In this particular case one has

$\frac{d D_{A}}{d r}=-\rho D_{A}$;

and the second order differential equation

$\frac{d^{2} D_{A}}{d r^{2}}=-\Phi_{00} D_{A}$.

It can be seen that in general $\rho$ has a value $r^{*}$ for which it vanishes and then change its sign. Then, the value of $D_{A}$ reaches an stationary value at $r^{*}$; which is a maximum, since $\Phi_{00}$ is positive when the usual energy conditions are considered to hold.

For this reason this notion of distance is not really a distance; since in particular it tends to zero when the source is at the farthest distance, i.e. at the beginning of the Universe; where $A \rightarrow 0$. But in any case it is a useful concept that can be employed to describe the behaviour of gravitational lenses.

\subsubsection{Future angular diameter distance and future area distance}

Let us also note that there is a notion of future angular diameter distance $d_{A}$, which is related to the angular diameter distance calculated by the emitter to reach an object of projected size $d l_{o}$ at the place of observer, perpendicular to the direction of the path of a photon. More concretely, one defines $d_{A}$ by the relation

$d l_{o}=d_{A} d \theta_{s} ;$

where $d \theta_{s}$ is the angle measured by a fiducial observer at the source with the same 4 -velocity as the source.

We can also generalize to the notion of future area distance; which is defined from:

$d A_{o}=\left|d_{+} d_{-}\right| d \Omega_{s}$

where $d A_{o}$ is the projected area of the object at the place of the observer, and $d \Omega_{s}$ is the solid angle generated at the source position, $d_{+}$and $d_{-}$are the future angular diameter distances along the principal directions of the area; and the future area distance $d_{\text {area }}$ is defined by

$d_{\text {area }}=\sqrt{\left|d_{+} d_{-}\right|}$.

Also in the case of a R-W geometry the future area distance coincides with the future angular diameter distance and so one can use $d_{A}\left(=d_{\text {area }}\right)$ for both notions.

We will discuss in section 4.4 the existing relation between the future area distance $d_{A}$ and the area distance $D_{A}$. For the moment, we will mention that in the most general case of an unresolved non-isotropic radiating source at redshift $z$ which emits with a luminosity $L\left(\theta_{s}, \phi_{s}\right)$ in the direction $\left(\theta_{s}, \phi_{s}\right)$, pointing out into the observer direction, one predicts that the flux $\mathscr{F}\left(\theta_{o}, \phi_{o}\right)$ measured by the observer is given by

$\mathscr{F}\left(\theta_{o}, \phi_{o}\right) d A_{o}=\frac{1}{(1+z)^{2}} L\left(\theta_{s}, \phi_{s}\right) d \Omega_{s} ;$

where the left hand side is the expression for the energy measured by the observer per unit time in the direction of the source $\left(\theta_{o}, \phi_{o}\right)$, collected in the surface element $d A_{o}$, and the right hand side is the expression for the energy emitted by the source per unit (local)time in the direction of the observer, in the solid angle $d \Omega_{s}$, with the corresponding redshift factor that takes into account the difference in local proper times, and the local energy measures. To clarify the notation, let us remark that if we call $L_{0}$ the total luminosity, and if the source where radiating isotropically, then one would have $L=L_{0} /(4 \pi)$.

Let us note that another way to write (78) is:

$d A_{o}=d_{\text {area }}^{2} d \Omega_{s}$ :

so that one finally has

$\mathscr{F}(\lambda, z)=\frac{L\left(\theta_{s}, \phi_{s}\right)}{(1+z)^{2} d_{\text {area }}^{2}(\lambda)} ;$

where we are emphasizing that the flux depends on the distance $\lambda$ and on the state of motion of the source, characterized by the redshift factor of expression (67).

Let us note that (82) is valid in a general spacetime and that what we call future angular diameter distance is what other authors call 'corrected luminosity distance'Perlick (2004): but we prefer our wording because it is more natural for the discussion of the reciprocity theorem that we present below.

\subsection{The Etherington theorem and the reciprocity corollary}

It is worthwhile to mention that the definition of area distance $D_{\text {area }}$ and future area distance $d_{\text {area }}$ have been presented for a general spacetime. For the general case there is a purely geometric result due to EtheringtonEtherington (1933) which establishes thatEllis (1971):

$d A_{o} d \Omega=(1+z)^{2} d A d \Omega_{s} ;$

which is known as the Etherington theorem. An interesting corollary of this result is derived from (73) and (81), since one can prove the relation between the area distances, namely

$d_{\text {area }}=(1+z) D_{\text {area }}$

This is a fundamental relation between the future area distance $d_{\text {area }}$ and the (observed) area distance $D_{\text {area }}$, also known as the reciprocity relation, which is only deduced from considerations of thin bundles connecting the source and the observer. 
Also, relation (84) is the main tool to prove the distanceduality relation, that we recall next.

\subsection{Luminosity distance and the distance duality-relation}

Let us return to the relation between the observed flux and the total luminosity of an unresolved emitter, shown in equation (82). This relation gives rise to the notion of luminosity distance $D_{L}$ which is defined in term of $\mathscr{F}\left(\theta_{o}, \phi_{o}\right)$ and $L\left(\theta_{s}, \phi_{s}\right)$ in a completely analogous way than in a flatspacetime, namely

$D_{L} \equiv \sqrt{\frac{L\left(\theta_{s}, \phi_{s}\right)}{\mathscr{F}\left(\theta_{o}, \phi_{o}\right)}} ;$

from which one can see that:

$D_{L}=(1+z) d_{\text {area }}$.

Although this definition has been presented for unresolved sources; estimates of distances from extended sources can be based on the same principle. In such cases the problem to infer distance for resolved or extended objects involves the consideration of the so called surface brightness. We will discuss these topics elsewhere.

\subsubsection{Distance-duality relation}

The combination of equation (86) together with the geometric relation established by the reciprocity theorem (84) gives as a corollary, the so called distance-duality relation:

$D_{L}(z)=(1+z)^{2} D_{\text {area }}$;

which links the luminosity distance with the area distance.

It is worthwhile to remark that this relation, valid for a general spacetime, has been derived just from a couple of assumptions, namely: that photons follow geodesics and that the number of photons is conserved. Since, the geodesic motion of photons is sustained by the undisputed equivalence principle, any observed deviations from the distance-duality relation would imply a non-conservation of the mean number of photon along null geodesicsBassett \& Kunz (2004).

\section{DIFFERENT NOTIONS OF MAGNIFICATIONS FOR GENERAL SPACETIMES}

\subsection{Angular magnification}

In a general spacetime the angular magnification $\mu$, which is normally addressed just as the magnification, is defined as the ratio between the solid angles subtended by the "lensed" and the "unlensed" image. When using the principal directions of the lensed image, one has the relations

$\delta \beta_{-}=(1-\kappa-\gamma) \delta \theta_{-}$,

and

$\delta \beta_{+}=(1-\kappa+\gamma) \delta \theta_{+} ;$

from which one can also deduce that

$\mu=\frac{1}{(1-\kappa)^{2}-\gamma^{2}}$; where $\gamma^{2}=\gamma_{1}^{2}+\gamma_{2}^{2}$ is the square of the module of the shear.

For most astrophysical situations one has $\kappa<<1$ and $\gamma<<1$. If we multiply, let us say, (88) by $\lambda$ we obtain

$d l_{-}=\lambda \delta \beta_{-}=\lambda(1-\kappa-\gamma) \delta \theta_{-}=D_{-} \delta \theta_{-}$,

and similarly with the + case. Then it is deduced that one can also express

$\mu=\left(\frac{\lambda}{D_{\text {area }}(\lambda)}\right)^{2}$

since the area distance in flat spacetime is just $\lambda$. This expression, valid for a general spacetime, coincides with the definition found in eq. (42) of the reference Perlick (2004).

\subsection{The intensity magnifications}

In this subsection we present the natural physical intensity magnification, that we will denote with $\tilde{\mu}$, and that we just call intensity magnification; but also it is convenient to introduce the astrophysical motivated cosmic intensity magnification, that we will denote with $\mu_{c}^{\prime}(z)$. The behavior of these intensity magnifications will be discussed below after the introduction of a couple of cosmic examples.

Let us denote again with $\mathscr{F}$ the observed flux of an unresolved object at distance $\lambda$ and relative motion determined by the redshift $z$, in a general spacetime. We will use $\mathscr{F}_{0}$ to denote the flux that one expects to collect from the same object at the same distance $\lambda$ in Minkowski spacetime with the same relative motion. Then, we define the intensity magnification $\tilde{\mu}$ by the quotient of these two fluxes, namely:

Definition 5.1 Intensity magnification:

$\tilde{\mu}(\lambda) \equiv \frac{\mathscr{F}(\lambda, z)}{\mathscr{F}_{0}(\lambda, z)}=\left(\frac{d_{\text {area } 0}(\lambda)}{d_{\text {area }}(\lambda)}\right)^{2} ;$

where, taking into account equation (82), one can see that actually $\tilde{\mu}$ turns out to depend only on the distance $\lambda$, and we use the subindex 0 to denote quantities in Minkowski spacetime.

It is probably worthwhile to remark that we are using here the same philosophy that one applies to the basic notion of gravitational lens in equation (1), where one compares angles in the sphere of directions between the lensed situation and the unlensed one (flat case).

It is crucial here to recall the reciprocity theorem, since it allows us to prove the following theorem, valid for a general spacetime:

Theorem 5.1 The intensity magnification coincides with the (standard angular) magnification; namely:

$\tilde{\mu}=\mu$.

Proof: We have seen above that a corollary of the Etherington theorem, presented in 4.4, is the reciprocity relation (84); so that in the expression for the intensity magnification above one can replace the future area distances by the observed area distances, and express

$\tilde{\mu}(\lambda)=\left(\frac{D_{\text {area0 }}(\lambda)}{D_{\text {area }}(\lambda)}\right)^{2}=\left(\frac{\lambda}{D_{\text {area }}(\lambda)}\right)^{2}=\mu ;$

where, we have used equation (92). 
It should be noted that this is a relation valid in a general spacetime which relates the here defined observable intensity magnification, with the standard angular magnification; instead the usual discussion is done relating the standard magnification with the non-observable luminosity distance as applied in spherically symmetric spacetimes. This is normally carried out in Robertson-Walker geometries that are spherically symmetric around every point of the spacetime. If the exact geometry of the Universe where represented by a Robertson-Walker geometries then it would be a matter of personal choice, whether one would like to use the concept of luminosity distance or of the observed flux; but we do know that the Universe is not homogeneous nor isotropic, and therefore it is important that theorem 5.1 is true for general spacetimes.

A very important point to remark is that astrophysicists normally think in the relation of the fluxes as a function of the observed redshift; so that from the observational point of view, it might become useful also to define

\section{Definition 5.2 Cosmic Intensity Magnification:}

$$
\begin{aligned}
\mu_{c}^{\prime}(z) & \equiv \frac{\mathscr{F}(z)}{\mathscr{F}_{\text {Milne }}(z)}=\left(\frac{1+z}{1+z}\right)^{4}\left(\frac{D_{A 0}(z)}{D_{A}(z)}\right)^{2} \\
& =\left(\frac{\lambda_{\text {Milne }}(z)}{D_{A}(z)}\right)^{2}
\end{aligned}
$$

where more specifically in this case one uses the flat Milne cosmological model, in order to have a relation $\lambda(z)$.

Here it should be remarked that although the definition of this intensity magnification also involves the quotient of the same fluxes; the definition (93) gives a different function because it involves a different parametrization of the scalars. For this same reason, the right hand side of (96) will not coincide with the corresponding cosmic magnification $\mu_{c}$ that it will be defined below in equation (109). In particular, since in the flat spacetime case we need a relation between the distance $\lambda$ and the redshift $z$, one makes use of the cosmological model given by the flat Milne Universe, that is recalled in the next subsection.

The reason we introduce the intensity magnification $\tilde{\mu}$ is because it is directly related to observations; since astrophysicists measure relative apparent magnitudes, as given byBradt (2004)

$m_{2}-m_{1}=-\frac{5}{2} \log \frac{\mathscr{F}_{2}}{\mathscr{F}_{1}}$

The collecting apparatus normally does not receive all the photons but those on a limited band; we assume here for the sake of simplicity in the discussion that all photons are collected; but one can generalize these concepts to the realistic situation.

To obtain intrinsic information, one can also use the so called absolute magnitude $M$; which is defined as the magnitude of the object if it were at the standard distance of 10 parsecs. The relation of the apparent magnitude and the absolute magnitude is given by

$$
\begin{aligned}
m-M & =-\frac{5}{2} \log \frac{\mathscr{F}(\lambda, z)}{\mathscr{F}\left(\lambda_{10}, z_{10}\right)}=5 \log \frac{D_{L}(\lambda, z)}{D_{L}\left(\lambda_{10}\left(z_{10}\right), z_{10}\right)} \\
& =-\frac{5}{2} \log \left(\frac{\mathscr{F}(\lambda, z)}{\mathscr{F}_{0}(\lambda, z)} \frac{\mathscr{F}_{0}(\lambda, z)}{\mathscr{F}_{0}\left(\lambda_{10}, z_{10}\right)} \frac{\mathscr{F}_{0}\left(\lambda_{10}, z_{10}\right)}{\mathscr{F}\left(\lambda_{10}, z_{10}\right)}\right) \\
& =-\frac{5}{2} \log \tilde{\mu}(\lambda)+5 \log \left(\frac{\lambda(1+z)^{2}}{\lambda_{10}}\right) ;
\end{aligned}
$$

where the first term in the last line corresponds to the first factor in the logarithm, the second term corresponds to the second factor in the logarithm, and we have neglected the contribution coming from the ratio $\frac{\mathscr{F}_{0}\left(\lambda_{10}\right)}{\mathscr{F}\left(\lambda_{10}\right)}$; since the cosmic incidence to the curvature for distances up to 10pc can be completely ignored.

We have used here the notation that $\mathscr{F}$ refers to the flux measured in the real (general) spacetime and $\mathscr{F}_{0}$ refers to the flux measured in the situation of empty (flat) spacetime.

It is important to emphasize that the left hand side of equation (98) is known as distance modulus, and that it can be completely expressed in terms of the gravitational lens optical scalars, since due to theorem 5.1 one can write

$m-M=-\frac{5}{2} \log \mu(\lambda)+5 \log \left(\frac{\lambda(1+z)^{2}}{\lambda_{10}}\right) ;$

where the magnification $\mu(\lambda)$ can be understood in terms of (90) or (92); which are valid for general spacetimes. Let us also note that the second term in the right hand side of (99) has kinematical information, and has no dependence on any possible cosmological background.

Let us now express the relation between magnifications when the redshift is used as the single variable to denote distance, as is normally done in cosmological studies. The previous expression involving the absolute magnification is now given by:

$$
\begin{aligned}
m-M= & -\frac{5}{2} \log \frac{\mathscr{F}(\lambda(z), z)}{\mathscr{F}\left(\lambda_{10}\left(z_{10}\right), z_{10}\right)} \\
= & 5 \log \frac{D_{L}(\lambda(z), z)}{D_{L}\left(\lambda_{10}\left(z_{10}\right), z_{10}\right)} \\
= & -\frac{5}{2} \log \left(\frac{\mathscr{F}(\lambda(z), z)}{\mathscr{F}_{\text {Milne }}(\lambda(z), z)} \frac{\mathscr{F}_{\text {Milne }}(\lambda(z), z)}{\mathscr{F}_{\text {Milne }}\left(\lambda_{10}\left(z_{10}\right), z_{10}\right)}\right. \\
& \left.\frac{\mathscr{F}_{\text {Milne }}\left(\lambda_{10}\left(z_{10}\right), z_{10}\right)}{\mathscr{F}\left(\lambda_{10}\left(z_{10}\right), z_{10}\right)}\right) \\
= & -\frac{5}{2} \log \mu_{c}^{\prime}(z)+5 \log \left(\frac{\lambda_{\text {Milne }}(z)(1+z)^{2}}{\lambda_{10}}\right)
\end{aligned}
$$

where again we have neglected the cosmic effects at the short distance of $10 \mathrm{pc}$. One can see that in this case the expression is given in terms of the intensity magnification, $\mu_{c}^{\prime}(z)$.

\section{ROBERTSON-WALKER SPACETIME AS A GRAVITATIONAL LENS}

\subsection{The fundamental concepts in the cosmological context}

It is important to remark that the basic notion of a gravitational lens is encoded in equation (1), in which the variation 
angle $\delta \beta^{a}$ refers to an unaffected spacetime, and therefore Minkowski; while the variation angle $\delta \theta^{b}$ refers to the real spacetime, in this case R-W. Then, when one considers this spacetime as a gravitational lens, this is not located at a particular position, it rather fills the whole spacetime. It is because of this reason that one has to be very careful with the interpretation of the optical scalars, as defined in equation (2).

We are thinking in the case of an observer within a R-W spacetime which, when studying the variations of deviation angles, he must relate the observed angle $\delta \theta^{a}$ with angle $\delta \beta^{a}$, when the source is at the 'same' distance; where as usual, the second angle refers to an observation where no gravitational effects are present. Therefore it is crucial to have a clear understanding of the notion of distance, that one must use. From all the notions of distance that are employed in the literature and that we have mentioned in section 4 , the most fundamental one, that requires the least structure is the geometric distance $\lambda$; since it only uses the information of the 4-velocity of the observer and the null geodesic coming from the source. In fact, it can be applied to any spacetime and any past directed null geodesic and is the dynamical parameter of the fundamental equation (23). Consequently, it constitutes the natural notion of distance in such context.

Then, for a source of size $\mathcal{X}$ at a distance $\lambda$ along the past null cone this observer would expect to see, in the absence of gravitational effects, that the source is subtending an angle $\delta \beta$ :

$\delta \beta=\frac{\mathcal{X}}{\lambda}$.

However, in R-W geometries equation (65) tells us that

$\mathcal{X}=A(t) f_{k}(\chi) \delta \theta$.

Then, from equation (66) one has the simple relation

$\delta \beta=\frac{D_{A}}{\lambda} \delta \theta$.

The relation between the observed angle $\delta \theta$ and $\delta \beta$ is understood as the lensing effect produced by the R-W cosmology. In the usual language of weak lensing this constitute a very peculiar lens since the whole spacetime acts as a lens which is not placed at a particular distance. Furthermore, in the R-W gravitational lens there is no bending angle due to the fact that it is an homogeneous and isotropic spacetime. In spite of this, one still has a non-trivial meaning of the optical scalars.

It is important to remark this fact because it is a source of confusion. For instance, the diagram appearing in figure 1 comes from the normal situation one encounters for a localized gravitational lens. However, in the case in which the whole spacetime is acting as a lens (which is not localized) the diagram must be understood in the conceptual way using the definitions of $\delta \beta$ and $\delta \theta$ as the angles measured with no lens, and with lens, respectively. This issue is completely missed in approaches based on the bending angle concept, as is normally done in textbooksSchneider et al. (1992, 2006).

Another essential point that one should also note is that equations (102) and (103) are exact equations valid also for large values of $\delta \theta$. This means that one can regard the $\mathrm{R}-\mathrm{W}$ lens in a non-perturbative way Frittelli et al. (2000a,b).

\subsection{The cosmic convergence and angular cosmic magnification}

Equation (103) is telling us that no cosmic shear is present, and that the cosmological convergence $\kappa_{c}$ can be deduced from

$\delta \beta=\left(1-\kappa_{c}\right) \delta \theta ;$

so that one has

$\kappa_{c}=1-\frac{D_{A}(\lambda)}{\lambda}$.

We emphasize again that the cosmic convergence $\kappa_{c}$ can not be defined using the standard approach to gravitational lenses bases on the bending angle concept.

In general, $D_{A}(\lambda)<\lambda$ holds for all values of $\lambda$ and for close objects one has that $\kappa_{c} \ll 1$. In fact, this is the common situation that one finds in the discussion of weak gravitational lenses in which the magnitudes of the optical scalars are always much smaller than the unit value and eq. (1) represents an approximation for small variations from chosen angular directions. However as indicated above, in the R-W geometry the cosmic convergence is exactly given by (105), which goes to the unit value as one approaches the initial cosmic singularity.

The behaviour of $\kappa_{c}$ close to the observer can be investigated taking an expansion of $D_{A}$ in terms of $\lambda$; we found:

$\kappa_{c}=\kappa_{c}^{(2)} \lambda^{2}+\kappa_{c}^{(3)} \lambda^{3}+\mathscr{O}\left(\lambda^{4}\right) ;$

where

$\kappa_{c}^{(2)}=\left.\frac{1}{3 !} \Phi_{00}\right|_{\lambda=0}$,

$\kappa_{c}^{(3)}=-\left.\frac{2}{4 !} \ell\left(\Phi_{00}\right)\right|_{\lambda=0}$.

From the previous discussion of angular magnification in general spacetimes, one can now see that the cosmic magnification $\mu_{c}$, is given by

$\mu_{c}=\frac{1}{\left(1-\kappa_{c}\right)^{2}}=\left(\frac{\lambda}{D_{A}(\lambda)}\right)^{2}$.

It is probably worthwhile to emphasize that the content of the previous equation coincides with equation (103); which as mentioned before it is an exact equation valid even for large values of $\delta \theta$.

When the previous theorem, on the relation between magnification and intensity magnification, is applied to the cosmological scenario, one obtains:

$\tilde{\mu}_{c}=\mu_{c}$.

Let us notice that $\mu_{c}$ diverges as the source is considered close to the initial singularity; which in turns indicates that $\kappa_{c}$ approaches the unit value, and therefore it can not be assumed to be small at large distances.

\subsection{Example 1: Milne Universe}

As a simple illustration of the family of cosmological R-W lenses we consider the simplest one which corresponds to the Milne Universe; which is the case of an empty cosmology, which is interpreted as the limit of vanishing density 
of an open dust model. For these reason, the whole Milne Universe, defined by the coordinates of the R-W metric, is identical from the point of view of the geometry to a portion of Minkowski spacetime. This geometry is characterized by $k=-1$ and $A(t)=c t$, since we are assuming $A$ has units of length; although we use units for which the universal constant $c$ is one. In terms of co-moving coordinates the relevant distances for the discussion of lenses are

$\lambda_{\text {Milne }}=\frac{1}{2} \frac{A^{2}\left(t_{0}\right)-A^{2}(t)}{A\left(t_{0}\right)}=A\left(t_{0}\right) z \frac{1+\frac{z}{2}}{(1+z)^{2}}$,

$D_{A}=\lambda$,

$D_{L}=\left(\frac{A\left(t_{0}\right)}{A(t)}\right)^{2} \lambda$

which then yield a vanishing cosmological convergence as expected:

$\kappa_{c}=0$,

$\mu_{c}=\tilde{\mu}_{c}=\mu_{c}^{\prime}=1$

\subsection{Example 2: Friedman Universes}

We concentrate in this subsection on the non-flat(4dimensional sense) Friedman Universes; in particular we will focus on the models that involve only dust, radiation and cosmological constant.

In general, analytical expressions for the optical quantities can not be obtained, however we content our self with the study of the behaviour in a neighbourhood of a fundamental observer.

The curvature component $\Phi_{00}$ and its derivative with respect to $\ell$ have the expressions:

$$
\begin{aligned}
\Phi_{00}= & \frac{4 \pi G}{c^{2}} \frac{A^{2}\left(t_{0}\right)}{A^{2}(t)}\left(\varrho(t)+\frac{P(t)}{c^{2}}\right) ; \\
\ell\left(\Phi_{00}\right)= & \frac{4 \pi G}{c^{3}} \frac{A^{3}\left(t_{0}\right)}{A^{3}(t)}\left[\frac{d \varrho}{d t}+\frac{1}{c^{2}} \frac{d P}{d t}\right. \\
& \left.-2 H(t)\left(\varrho(t)+\frac{P(t)}{c^{2}}\right)\right] ;
\end{aligned}
$$

where $G$ is the gravitational constant, $c$ is the speed of light, $\varrho(t)$ and $P(t)$ the matter density and pressure of the cosmic fluid respectively and $H(t)$ is the Hubble's rate of expansion defined as

$H(t) \equiv \frac{1}{A(t)} \frac{d A}{d t}$.

For convenience in this section and in all our final results we show the appearance of the Universal constants.

We can write all quantities of the fluids and the geometric functions, as for example $H(t)$, in terms of the so-called density parameters $\left(\Omega_{m}, \Omega_{r}, \Omega_{\Lambda}\right)$ and the critical density $\varrho_{c r}$ :

$$
\begin{aligned}
\varrho(t) & =\varrho_{c r}\left(\Omega_{m} \frac{A^{3}\left(t_{0}\right)}{A^{3}(t)}+\Omega_{r} \frac{A^{4}\left(t_{0}\right)}{A^{4}(t)}+\Omega_{\Lambda}\right), \\
\frac{P(t)}{c^{2}} & =\varrho_{c r}\left(\frac{\Omega_{r}}{3} \frac{A^{4}\left(t_{0}\right)}{A^{4}(t)}-\Omega_{\Lambda}\right),
\end{aligned}
$$

$\frac{H^{2}(t)}{H^{2}\left(t_{0}\right)}=-\frac{k c^{2}}{H\left(t_{0}\right)^{2} A(t)^{2}}+\Omega_{m} \frac{A^{3}\left(t_{0}\right)}{A^{3}(t)}+\Omega_{r} \frac{A^{4}\left(t_{0}\right)}{A^{4}(t)}+\Omega_{\Lambda}$.

The evaluation of $A\left(t_{0}\right)$ is through the equation

$1=-\frac{k c^{2}}{H\left(t_{0}\right)^{2} A\left(t_{0}\right)^{2}}+\Omega_{r}+\Omega_{m}+\Omega_{\Lambda} ;$

which is just the evaluating of (121) at the present time. In the case $k=0$ one has a freedom in the value of $A\left(t_{0}\right)$ which can be taken as $A\left(t_{0}\right)=\frac{c}{H\left(t_{0}\right)}$.

This yields,

$$
\begin{aligned}
\Phi_{00} & =\frac{4 \pi G \varrho_{c r}}{c^{2}} \frac{A^{5}\left(t_{0}\right)}{A^{5}(t)}\left(\Omega_{m}+\frac{4}{3} \Omega_{r} \frac{A\left(t_{0}\right)}{A(t)}\right), \\
\ell\left(\Phi_{00}\right) & =-\frac{4 \pi G \varrho_{c r}}{c^{3}} \frac{A^{6}\left(t_{0}\right)}{A^{6}(t)} H(t)\left(5 \Omega_{m}+8 \Omega_{r} \frac{A\left(t_{0}\right)}{A(t)}\right) .
\end{aligned}
$$

Then, the cosmological convergence $\kappa_{c}$, near the observer, behaves in the following way:

$$
\begin{aligned}
\kappa_{c}(\lambda) & =\frac{4 \pi G \varrho_{c r}}{3 ! c^{2}}\left(\Omega_{m}+\frac{4}{3} \Omega_{r}\right) \lambda^{2} \\
& +\frac{8 \pi G \varrho_{c r}}{4 ! c^{3}} H_{0}\left(5 \Omega_{m}+8 \Omega_{r}\right) \lambda^{3}+\mathscr{O}\left(\lambda^{4}\right) .
\end{aligned}
$$

It is probably interesting to remark that the only optical scalar that appears in the gravitational lens study of the $\mathrm{R}-\mathrm{W}$ geometry is independent of the cosmological constant contribution $\Omega_{\Lambda}$.

\subsection{Typical values for the cosmic convergence and magnification functions}

The last examples allow to estimate the effect of the R-W spacetimes as a gravitational lens in a simple way. In this section we present the values of the cosmic convergence and magnification functions for a couple of representative cosmic models; one based on the data of the Planck Collaboration and the other just coming from the primordial nucleosynthesis calculations.

\subsubsection{Values from Planck Collaboration}

The following values, used and reported by the Planck Collaboration in reference Ade et al. (2014), were employed to compute the different quantities associated to the lens effect; that we show below in table 1 .

$$
\begin{aligned}
\Omega_{m} & =0,314 \pm 0,020, \\
\Omega_{\Lambda} & =0,686 \pm 0,020, \\
H_{0} & =67,4 \pm 1,4 \frac{\mathrm{km}}{\mathrm{s} \cdot \mathrm{Mpc}}, \\
k & =0 .
\end{aligned}
$$

In the above list we have also included the corresponding values for $z_{0}$; i.e. the value that would take the redshift if the universe where the flat Milne spacetime, for a given geometric distance $\lambda$.

Then, it is curious that while the convenient astrophysical intensity magnification $\mu_{c}^{\prime}$ shows a fainter behavior for this range of redshift values, in qualitative agreement with 
Table 1. Planck $\Lambda$ CDM model

\begin{tabular}{cccccc}
$\lambda[\mathrm{Mpc}]$ & $z_{0}$ & $z$ & $\kappa_{c}$ & $\tilde{\mu}_{c}=\mu_{c}$ & $\mu_{c}^{\prime}$ \\
\hline 43.95 & 0.010 & 0.01 & $7.85 \times 10^{-6}$ & 1.00002 & 0.99481 \\
169.55 & 0.040 & 0.04 & 0.00013 & 1.00025 & 0.98043 \\
395.164 & 0.103 & 0.10 & 0.00078 & 1.00157 & 0.95642 \\
591.27 & 0.167 & 0.16 & 0.00200 & 1.00401 & 0.93785 \\
788.85 & 0.245 & 0.23 & 0.00411 & 1.00826 & 0.92189 \\
935.68 & 0.314 & 0.29 & 0.00647 & 1.01307 & 0.91231 \\
1179.06 & 0.459 & 0.41 & 0.01266 & 1.02581 & 0.90235 \\
1311.31 & 0.561 & 0.49 & 0.01776 & 1.03649 & 0.90127 \\
1474.92 & 0.722 & 0.61 & 0.02668 & 1.05557 & 0.90624 \\
1735.04 & 1.131 & 0.88 & 0.05107 & 1.11055 & 0.93905 \\
1915.09 & 1.680 & 1.18 & 0.08261 & 1.18821 & 0.99991 \\
2121.16 & 3.629 & 1.85 & 0.15795 & 1.41034 & 1.19321
\end{tabular}

Table 2. Baryonic Low density model

\begin{tabular}{cccccc}
$\lambda[\mathrm{Mpc}]$ & $z_{0}$ & $z$ & $\kappa_{c}$ & $\tilde{\mu}_{c}=\mu_{c}$ & $\mu_{c}^{\prime}$ \\
\hline 41.04 & 0.010 & 0.01 & $1.06 \times 10^{-6}$ & 1.00000 & 1.00021 \\
157.07 & 0.040 & 0.04 & 0.00002 & 1.00003 & 1.00087 \\
361.12 & 0.100 & 0.10 & 0.00010 & 1.00020 & 1.00223 \\
560.36 & 0.170 & 0.17 & 0.00028 & 1.00057 & 1.00391 \\
748.05 & 0.249 & 0.25 & 0.00059 & 1.00118 & 1.00592 \\
884.86 & 0.319 & 0.32 & 0.00093 & 1.00187 & 1.00776 \\
1114.46 & 0.467 & 0.47 & 0.00189 & 1.00378 & 1.01191 \\
1232.04 & 0.565 & 0.57 & 0.00266 & 1.00534 & 1.01483 \\
1379.10 & 0.720 & 0.73 & 0.00409 & 1.00824 & 1.01969 \\
1619.77 & 1.121 & 1.15 & 0.00872 & 1.01767 & 1.03341 \\
1793.70 & 1.684 & 1.77 & 0.01701 & 1.03491 & 1.05540 \\
1913.14 & 2.503 & 2.77 & 0.03215 & 1.06754 & 1.09353
\end{tabular}

observationsRiess et al. (1998); Perlmutter et al. (1999); Riess et al. (2004), the more physical intensity magnification $\tilde{\mu}_{c}$ implies a brighter behavior for the same range. This emphasizes the fact that the notions of fainter or brighter, which are very common in the observational language, are rather relative; since they depend on what setting one is assigning these concepts.

\subsubsection{Values from primordial nucleosynthesis calculations}

Here we consider the set of parameters that come just from the primordial nucleosynthesis calculations and independent observation of the Hubble parameter, and others, that are shown next.

$\Omega_{m}=0,042569$,

$\Omega_{r}=4.7647 \times 10^{-5}$

$\Omega_{\Lambda}=0$,

$H_{0}=72 \frac{\mathrm{km}}{\mathrm{s} \mathrm{Mpc}}$,

$$
k=-1 \text {. }
$$

In table 2 below, we present the result of the calculations for the different quantities associated to the cosmic lens effects, using these values.

As expected, this model which is practically devoid of matter, presents a behaviour with small deviations from the trivial flat Milne Universe. We also note that the intensity magnification $\mu_{c}^{\prime}$ is always greater than one in the whole range of the affine parameter.

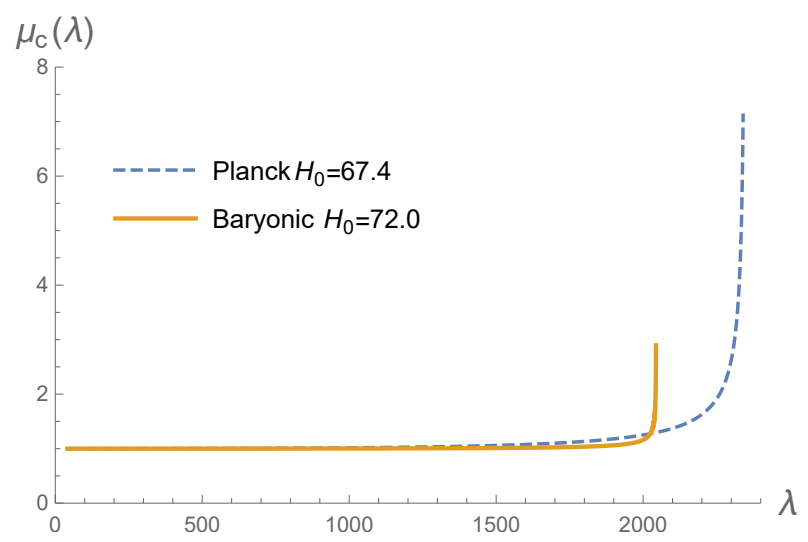

Figure 3. Since in general $\lambda>D_{A}(\lambda)$ the cosmic magnification is greater than one and divergent near of the initial singularity, except in the trivial case of flat spacetime.

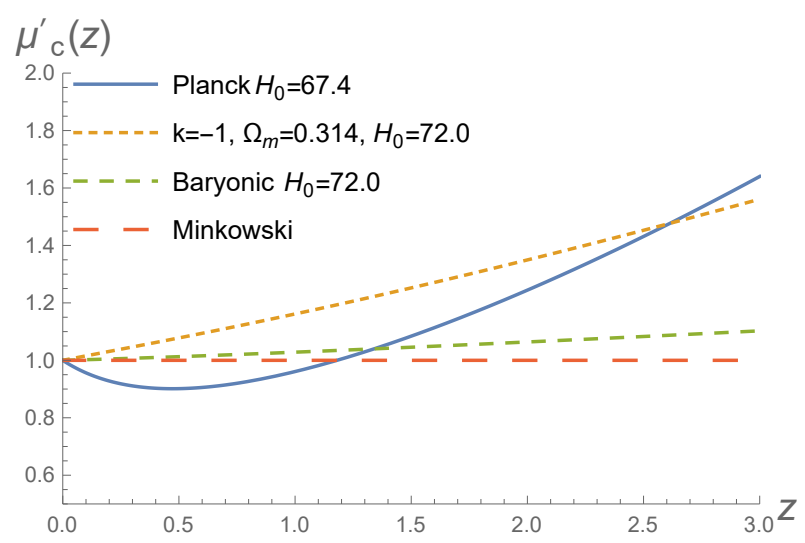

Figure 4. The astrophysical magnification $\mu_{c}^{\prime}(z)$ is shown for the Planck and Baryonic models. Milne Universe and an open universe with only matter content $\Omega_{m}=0.314$ are also shown.

\subsubsection{Plots for these previous models}

In this subsection we present a graph illustrating the behaviour of the physical intensity magnification $\tilde{\mu}_{c}=\mu_{c}$ in terms of the geometric distance, together with the graph of the intensity magnification $\mu_{c}^{\prime}(z)$; as used in astrophysical works. One can see in these graphs how the notion of 'brighter' and/or 'fainter' depends in the way the observational data is studied.

\section{THE PRESENCE OF AN ADDITIONAL LENS OVER THE COSMOLOGY}

\subsection{The main exact equation}

In the previous section we have shown that a $\mathrm{R}-\mathrm{W}$ spacetime can be thought as a weak gravitational lens. In the current section we will deal with additional lenses on the geometry. We mean by this an alteration of the completely homogeneous and isotropic cosmological background where the size of the additional lens must be much smaller that the cosmological scale, since otherwise it would change the cosmology one is discussing. This means that normally the time of flight of photons trough the additional lens would 
be considered small compared with the total time of flight. By time of flight we mean more precisely the lapse of the geometric affine distance.

In order to compute distortions due to the presence of inhomogeneities we will consider the exact equation (23) with the source of curvature component decomposed in terms of the cosmological background and the lens contribution, in the following way:

$\ell(\ell(\mathcal{X}))=-\left(Q_{B}+Q_{L}\right) \mathcal{X}$

where we have included the subindex $B$ to denote intrinsic quantities of the background and $L$ to denote quantities associated to the additional lens. In other words, the components of the curvature appearing in $Q$ in equation (23) is now expressed as

$Q=Q_{B}+Q_{L}$.

In particular $Q_{L}$ is the matrix containing the difference from the background curvature which is given in terms of the scalars:

$Q_{L}=\left(\begin{array}{cc}\Phi_{00}^{L} & \Psi_{0}^{L} \\ \bar{\Psi}_{0}^{L} & \Phi_{00}^{L}\end{array}\right)$

As mentioned in the introduction, it is worthwhile to emphasized that in the decomposition of the total curvature in terms of the background plus a lens term, it is not assumed that the additional term must be small in any sense. That is we are not assuming at this stage any kind of perturbation; it is just the representation of an exact geometry, without perturbation, in terms of a decomposition with respect to a chosen background. This becomes more clear when we fix the issue of gauges in the next subsection.

\subsection{A treatment of the main equation without room for gauges}

As usual, referring quantities with respect to a background, lead us to deal with the delicate issues associated to the gauge freedom. So, with the aim to clarify the way in which equation (136) should be understood, it is pertinent to present a short explanation before tackling the problem of solving the equation.

A gauge choice in the sense of Stewart \& Walker (1974); Stewart (1990); Bardeen (1980) is a correspondence between a fiducial background (namely a R-W one) and the real inhomogeneous spacetime. In the context of cosmological perturbations the problem of how to specify this map appropriately has been called the "fitting problem"Ellis \& Stoeger (1987). A good understanding of the way in which one fully specifies the gauge is crucial at the moment to interpret tensor fields quantities that are regarded as departure from the backgroundEllis \& Bruni (1989).

In the simpler case of weak lensing over a flat background, the meaning of $Q_{L}$ is always well defined since the curvature is gauge invariant in such case. It is because of this specific fact that in referenceGallo \& Moreschi (2011) one prefers to express the optical scalar in terms of the curvature.

The discussion of gauge invariance is subtle, and one should have a clear picture of the geometrical and physical framework one is using. For example in references Stewart \& Walker (1974); Stewart (1990) they discuss the notion of identification gauge invariant(i.g.i) quantities. Then, since i.g.i. quantities should vanish on the background, one would deduce that while $\Psi_{0}^{L}$ is i.g.i, the curvature scalar $\Phi_{00}^{L}$ is not. However their treatment does not apply to our setting, as we now explain.

In our work, all these gauge issues are solved when the curvature components $Q_{B}$ is taken from observation. The measurements we have in mind are all the cosmological observations, as for example Plack's CMB measurement, or the supernovae observations, mentioned in references Perlmutter et al. (1999); Riess et al. (1998), where researchers used the cosmological observation of luminosity and redshift of supernovae to fit a Robertson-Walker geometry to the actual spacetime. After the parameters determining the RobertsonWalker geometry are fixed from observation, and the direction and magnitude of our velocity with respect the CMB is determined; the RW geometry is completely determined in our past null cone; this means metric, connection and curvature. In other words, the RW cosmic background is uniquely defined on our past null cone in terms of observations; and therefore there is no gauge freedom allowed in this respect.

Then, since (135) is an exact equation in terms of the exact geodesics, and $Q$ is therefore unambiguously defined; one concludes that $Q_{L}$ also does not have room for any gauge freedoms. In our framework $Q_{L}$ is the contribution to the curvature that is needed to explain the gravitational lensing observations. That is, both curvature terms $Q_{B}$ and $Q_{L}$ are determined from independent set of observations; where no gauge freedoms intervene.

Since one has at hand the back ground RW geometry, one can also decompose the exact metric in terms of $g^{a b}=$ $g_{B}^{a b}+h^{a b}$; where $g_{B}^{a b}$ is the metric of the RW geometry, and $h^{a b}$ is the needed tensor to complete the metric. Therefore we have at our disposal the complete RW geometry, including metric and solutions of geodesic equations; that we will use next. We should probably remark that at this stage we do not assume a particular form for $h^{a b}$, since we only require to describe the observed $Q_{L}$.

\subsection{The technique for solving the main equation}

Let us mention again that equation (135) is exact, and therefore it uses the exact geodesics; however, in order to find solutions it is more convenient to carryout the calculation, at the linear order in the curvature $Q_{L}$, using the background geodesics. This forces us to estimate the magnitude of the error involved in this procedure. So, in the following we present an estimate of this error.

Let us begin denoting by $\boldsymbol{\mathcal { X }}_{E}$ the exact solution along the exact null geodesic, which we indicate as $x_{E}^{\alpha}(\lambda)$. Then, the integral version of equation (135) is

$$
\begin{aligned}
& \boldsymbol{\mathcal { X }}_{E}(\lambda)=\ell\left(\boldsymbol{\mathcal { X }}_{E}\right) \|_{0}\left(\lambda-\lambda_{0}\right) \\
& \quad-\int_{\lambda_{0}}^{\lambda} \int_{\lambda_{0}}^{\lambda^{\prime}}\left(Q_{B}+Q_{L}\right)\left(x_{E}^{\alpha}\left(\lambda^{\prime \prime}\right)\right) \mathcal{X}_{E}\left(x_{E}^{\alpha}\left(\lambda^{\prime \prime}\right)\right) d \lambda^{\prime \prime} d \lambda^{\prime} .
\end{aligned}
$$

Now, let us also consider the above expression computed along the geodesics of the background which we will indicate 
by $x^{\alpha}(\lambda)$; we mean

$$
\begin{aligned}
\mathcal{X}(\lambda)= & \ell(\mathcal{X}) \|_{0}\left(\lambda-\lambda_{0}\right) \\
& -\int_{\lambda_{0}}^{\lambda} \int_{\lambda_{0}}^{\lambda^{\prime}}\left(Q_{B}+Q_{L}\right)\left(x^{\alpha}\left(\lambda^{\prime \prime}\right)\right) \mathcal{X}\left(x^{\alpha}\left(\lambda^{\prime \prime}\right)\right) d \lambda^{\prime \prime} d \lambda^{\prime} ;
\end{aligned}
$$

where in order to differentiate the result from the exact one, we have omitted the subindex $E$ for the vector $\mathcal{X}\left(x^{\alpha}(\lambda)\right)$ which contains the components of the deviation vector (15). To simplify the notation we are using the same symbols for the curvature components but it should be remarked that there is a slight change in the null tetrads. The relation between the exact geodesics and those of the background will be denoted as $x_{E}^{\alpha}(\lambda)-x^{\alpha}(\lambda) \equiv \delta x^{\alpha}(\lambda)$.

Then, we will consider the difference $\delta \mathcal{X}\left(x^{\alpha}(\lambda)\right) \equiv$ $\boldsymbol{\mathcal { X }}_{E}\left(x_{E}^{\alpha}(\lambda)\right)-\mathcal{X}\left(x^{\alpha}(\lambda)\right)$ between the exact result, namely equation (138) and the alternative procedure given by equation (139) and we will show that such difference is lower order than $\mathcal{X}(\lambda)$. Defining $\boldsymbol{\delta} \mathcal{X}$ in this way it is implicit that we are using the same procedure to define the auxiliary vector $m$ and $\bar{m}$ for both geodesics. Notice that $\delta$ quantities are of order $\mathscr{O}\left(Q_{L}\right)$. Explicitly, we have

$$
\begin{aligned}
\delta \mathcal{X}= & -\int_{\lambda_{0}}^{\lambda} \int_{\lambda_{0}}^{\lambda^{\prime}}\left(Q_{B}+Q_{L}\right)\left(x_{E}^{\alpha}\left(\lambda^{\prime \prime}\right)\right) \mathcal{X}_{E}\left(x_{E}^{\alpha}\left(\lambda^{\prime \prime}\right)\right) d \lambda^{\prime \prime} d \lambda^{\prime} \\
& +\int_{\lambda_{0}}^{\lambda} \int_{\lambda_{0}}^{\lambda^{\prime}}\left(Q_{B}+Q_{L}\right)\left(x^{\alpha}\left(\lambda^{\prime \prime}\right)\right) \mathcal{X}\left(x^{\alpha}\left(\lambda^{\prime \prime}\right)\right) d \lambda^{\prime \prime} d \lambda^{\prime} \\
= & -\int_{\lambda_{0}}^{\lambda} \int_{\lambda_{0}}^{\lambda^{\prime}}\left[Q_{B}\left(x_{E}^{\alpha}\left(\lambda^{\prime \prime}\right)\right)-Q_{B}\left(x^{\alpha}\left(\lambda^{\prime \prime}\right)\right)\right] \mathcal{X}\left(x^{\alpha}\left(\lambda^{\prime \prime}\right)\right) d \lambda^{\prime \prime} d \lambda^{\prime} \\
& -\int_{\lambda_{0}}^{\lambda} \int_{\lambda_{0}}^{\lambda^{\prime}}\left[Q_{L}\left(x_{E}^{\alpha}\left(\lambda^{\prime \prime}\right)\right)-Q_{L}\left(x^{\alpha}\left(\lambda^{\prime \prime}\right)\right)\right] \mathcal{X}\left(x^{\alpha}\left(\lambda^{\prime \prime}\right)\right) d \lambda^{\prime \prime} d \lambda^{\prime} \\
& -\int_{\lambda_{0}}^{\lambda} \int_{\lambda_{0}}^{\lambda^{\prime}}\left(Q_{B}+Q_{L}\right)\left(x_{E}^{\alpha}\left(\lambda^{\prime \prime}\right)\right) \boldsymbol{\delta} \mathcal{X}\left(x^{\alpha}\left(\lambda^{\prime \prime}\right)\right) d \lambda^{\prime \prime} d \lambda^{\prime} ;
\end{aligned}
$$

where the initial derivatives of $\mathcal{X}$ do not appear since, they represent the values of the observations, that are the same for both calculations; in other words, we are here comparing two calculation for the optical scalars in terms of curvature $Q_{L}$ that is determined by the existence of the lens. Also, let us remember that in weak lensing computations one is interested in the first order effects of the curvature $Q_{L}$. We will see below that the expressions for $\mathcal{X}$ will involve terms up to order $\mathscr{O}\left(Q_{B} Q_{L}\right)$. In any case, one can see that just keeping linear terms in $Q_{L}$, from the previous expression one obtains:

$$
\begin{aligned}
\boldsymbol{\delta} \mathcal{X}= & -\int_{\lambda_{0}}^{\lambda} \int_{\lambda_{0}}^{\lambda^{\prime}} \\
& {\left[Q_{B}\left(x_{E}^{\alpha}\left(\lambda^{\prime \prime}\right)\right)-Q_{B}\left(x^{\alpha}\left(\lambda^{\prime \prime}\right)\right)\right] \mathcal{X}\left(x^{\alpha}\left(\lambda^{\prime \prime}\right)\right) d \lambda^{\prime \prime} d \lambda^{\prime} } \\
& -\int_{\lambda_{0}}^{\lambda} \int_{\lambda_{0}}^{\lambda^{\prime}} Q_{B}\left(x^{\alpha}\left(\lambda^{\prime \prime}\right)\right) \boldsymbol{\delta} \mathcal{X}\left(x^{\alpha}\left(\lambda^{\prime \prime}\right)\right) d \lambda^{\prime \prime} d \lambda^{\prime} \\
& +\mathscr{O}\left(Q_{L}^{2}\right)
\end{aligned}
$$

where only $Q_{B}$ appears explicitly.

First of all, let us note that $\boldsymbol{\delta} \mathcal{X}$ appears only in the second term of the right hand side; so that a first estimate of it is just the first term. Then, let us also see that the exact null vector $\ell$ can always be expressed with respect to an appropriately chosen null geodesic vector of the background with a slight correcting conformal factor. To this, let us add that although $Q_{B}$ refers to an homogeneous spacetime, both geodesics are different in the lens and therefore have different affine length in the lens. Both effects are of the same nature and can be estimated by the difference in the affine length of the geodesics inside the lens. Let us call this difference $\delta \lambda_{L}$. If $\alpha$ is the deviation angle for a typical geodesics; then one could estimate $\delta \lambda_{L}$ from $\delta \lambda_{L}=\delta_{l}(1-\cos (\alpha))$ where $\delta_{l}$ is the characteristic radial size of the lens. Let us apply this to a spherical distribution of matter, and to a photon trajectory with impact parameter $J$, such that a quantity $M$ of matter is contained in a sphere of radius $J$; then one would have $\alpha=\frac{4 M}{J}$, using geometric units. For a typical $\alpha \ll 1$ one would have $\delta \lambda_{L} \cong \delta_{l} \frac{\alpha^{2}}{2}=\delta_{l} \frac{8 M^{2}}{J^{2}}$. Proceeding with the calculation we now estimate

$$
\begin{aligned}
-\int_{\lambda_{0}}^{\lambda} \int_{\lambda_{0}}^{\lambda^{\prime}} & {\left[Q_{B}\left(x_{E}^{\alpha}\left(\lambda^{\prime \prime}\right)\right)-Q_{B}\left(x^{\alpha}\left(\lambda^{\prime \prime}\right)\right)\right] \mathcal{X}\left(x^{\alpha}\left(\lambda^{\prime \prime}\right)\right) d \lambda^{\prime \prime} d \lambda^{\prime} } \\
& \cong\left(\lambda_{s}-\lambda_{l}\right) Q_{B}\left(\lambda_{l}\right) \mathcal{X}\left(x^{\alpha}\left(\lambda_{l}\right)\right) \delta \lambda_{L} \\
& =\left(\lambda_{s}-\lambda_{l}\right) Q_{B}\left(\lambda_{l}\right) \mathcal{X}\left(x^{\alpha}\left(\lambda_{l}\right)\right) \delta_{l} \frac{8 M^{2}}{J^{2}} .
\end{aligned}
$$

Typically we will take $J \approx \frac{\delta_{l}}{2}$. Let us apply this to a typical situation in which the lens has a radshift $z_{l}=0.06$, while the lens has redshift of $z_{s}=0.33$, for a galaxy we take $\delta_{l} \sim$ 200kpc and $M \sim 10^{-8} \mathrm{Mpc}$; while for a cluster of galaxies we take $\delta_{l} \sim 1 \mathrm{Mpc}$ and $M \sim 10^{-5} \mathrm{Mpc}$. Then we obtain for a galaxy $\frac{\delta \mathcal{X}}{\mathcal{X}} \sim 10^{-11}$, and for a cluster of galaxies $\frac{\delta \mathcal{X}}{\mathcal{X}} \sim 10^{-6}$.

We conclude then that using the null geodesics of the cosmological background to carry out the calculation involves deviations of the values that are completely negligible; and therefore it is safe to use this technique.

After corroborating the feasibility of the path integration technique, let us concentrate in the details of how to handle the algebra of the computation. Let us recall that from the physical point of view, one integrates equation (135) from the position of the observer along the past null cone. Therefore, although one could start with finite values of $\mathcal{X}$, we are only interested on initial conditions that have the information of the null cone, and so, our $\mathcal{X}$ 's start with a diverging behavior; dictated by the observing angle. For this reason we look for solutions of the form

$\mathcal{X}=M D_{A} \mathcal{V}_{0}$

where $M$ is a well behaved $2 \times 2$ square matrix and $\mathcal{V}_{0}$ has the same meaning than before; it is the observed angle subtended by the image: $\mathcal{V}_{0} \equiv \delta \theta$. This ansatz is reminiscent of the solution (65), since one recognizes the background solution $\mathcal{X}_{B}=D_{A} \mathcal{V}_{0}$.

It is probably interesting to point out that in the weak lensing regime, the matrix $M$ can be related to the optical scalars matrix $\mathcal{A}$ mentioned in (1); since taking the relation $\lambda \delta \beta=\mathcal{X}$ one has

$\mathcal{A}=\frac{D_{A}}{\lambda} M=\left(1-\kappa_{c}\right) M$.

In other words, the total gravitational lens combines the 
global cosmic convergence $\kappa_{c}$ with the 'local' lensing effects contained in $M$.

In writing equation (144) we should note that we have change the basis in which the matrix $\mathcal{A}$ is represented; since the matrix $\mathcal{A}_{b}^{a}$ of equations (1) and (2) refer to a base in the sphere of observed directions, while $\mathcal{A}$ in (144) refers to the complex components of $\mathcal{X}$. In order to relate both representations it is necessary to give an explicit relation of the complex vectors $m$ and $\bar{m}$ in terms of directions that can be expressed in turn in terms of directions in the sphere of observation. To fix ideas, let us consider the expression (27) of the space line element and consider the beam of light is coming from the positive $y$ direction, of a conformal Cartesian frame. Then, at any point we have at our disposal an orthonormal frame which respects the conformal Cartesian frame directions, and we express the null tetrad adapted to the incoming null geodesic by:

$$
\begin{aligned}
l^{a} & =(1,0,-1,0), \\
m^{a} & =\frac{1}{\sqrt{2}}(0,1,0, i), \\
\bar{m}^{a} & =\frac{1}{\sqrt{2}}(0,1,0,-i), \\
n^{a} & =\frac{1}{2}(1,0,1,0) ;
\end{aligned}
$$

which is a slight different notation that the one used in Gallo \& Moreschi (2011).

Now, in order to compare with the usual expressions for the lens scalars $\kappa, \gamma_{1}$ and $\gamma_{2}$, let us recall that they are defined via the relations (1) and (2); but since it is a linear relation, one can relate the deviation vectors by the same matrix, namely

$\varsigma_{s}^{i}=A_{j}^{i} \varsigma_{o}^{j}$

where $\left\{\varsigma_{s}^{i}, \varsigma_{o}^{i}\right\}$ are the spatial vector associated with $\left\{\varsigma_{s}, \varsigma_{o}\right\}$ respectively. In this expression, it is needed to determine the meaning of the indices $(i, j)$ of the two dimensional space of the images. For the purpose of connecting the natural Cartesian orientation we identify the first component of the two dimensional space with the $x$ direction, and the second component with the $z$ one. We need then, to know the components of the spatial vectors $\varsigma_{o}^{a}$ generated by $\varsigma_{o}$ and similarly by $\varsigma_{s}$ in a Cartesian like coordinate system. In the case of $\varsigma_{o}^{a}$, it is given by

$$
\begin{aligned}
\varsigma_{o}^{a} & =\varsigma_{o} \bar{m}^{a}+\bar{\varsigma}_{o} m^{a} \\
& =\frac{1}{\sqrt{2}}\left(\varsigma_{o}(0,1,0,-i)+\bar{\varsigma}_{o}(0,1,0, i)\right) \\
& =\frac{1}{\sqrt{2}}\left(0,\left(\varsigma_{o}+\bar{\varsigma}_{o}\right), 0, i\left(\bar{\varsigma}_{o}-\varsigma_{o}\right)\right) \\
& =\frac{2}{\sqrt{2}}\left(0, \varsigma_{o R}, 0, \varsigma_{o I}\right) ;
\end{aligned}
$$

and a similar expression is obtained for $\varsigma_{s}^{a}$.

Therefore, by replacing into eq.(146), we obtain

$$
\begin{aligned}
\varsigma_{s R} & =\left(1-\kappa-\gamma_{1}\right) \varsigma_{o R}-\gamma_{2} \varsigma_{o I}, \\
\varsigma_{s I} & =-\gamma_{2} \varsigma_{o R}+\left(1-\kappa+\gamma_{1}\right) \varsigma_{o I}
\end{aligned}
$$

which has a direct physical interpretation in the plane of the image, since the real component represents horizontal direction and the imaginary component the vertical one.
The equation satisfied by $M$ is found from equation (135):

$$
\begin{aligned}
\ell\left(\ell\left(M D_{A} \mathcal{V}_{0}\right)\right) & =\ell\left(\ell(M) D_{A} \mathcal{V}_{0}+M \ell\left(D_{A} \mathcal{V}_{0}\right)\right) \\
& =\ell(\ell(M)) D_{A} \mathcal{V}_{0}+2 \ell(M) \ell\left(D_{A} \mathcal{V}_{0}\right) \\
& +M \ell\left(\ell\left(D_{A} \mathcal{V}_{0}\right)\right) \\
& =\left(\ell(\ell(M))-2 \ell(M) P_{B}\right) D_{A} \mathcal{V}_{0} \\
& -M Q_{B} D_{A} \mathcal{V}_{0} \\
& =-\left(Q_{B}+Q_{L}\right) M D_{A} \mathcal{V}_{0}
\end{aligned}
$$

which simplifies to

$\ell(\ell(M))-2 \ell(M) P_{B}+Q_{L} M=0$.

Now, using the fact that for $\mathrm{R}-\mathrm{W}$ geometries one has the following important relation

$P_{B}=\mathbb{I} \rho=-\mathbb{I} \frac{\ell\left(D_{A}^{2}\right)}{2 D_{A}^{2}} ;$

one arrives to the final equation:

$\ell\left(D_{A}^{2} \ell(M)\right)+Q_{L} D_{A}^{2} M=0$.

\subsection{Solving the matrix equation}

In order to proceed to the integration of equation (153) the initial conditions must be specified. One can see that the appropriate ones for our problem are:

$$
\begin{aligned}
\left.\left(D_{A}^{2} \ell(M)\right)\right|_{r=0} & =0, \\
\left.M\right|_{r=0} & =\mathbb{I} .
\end{aligned}
$$

Using the first initial condition we obtain

$\ell(M)=-\frac{1}{D_{A}^{2}} \int_{0}^{r} Q_{L} D_{A}^{2} M d r^{\prime} ;$

while in the next step we found

$M=\mathbb{I}-\int_{0}^{r_{s}}\left(\frac{1}{D_{A}^{2}} \int_{0}^{r^{\prime}} Q_{L} D_{A}^{2} M d r^{\prime \prime}\right) d r^{\prime} ;$

where $r_{s}$ denotes the position of the emitting source.

Let us remember that the affine parameter $r$ takes negative values when it approaches to the source; due to this fact we prefer to use the normalized affine distance $\lambda$ to parametrize the geodesic. With this choice we rewrite the last equation to

$M=\mathbb{I}-\int_{0}^{\lambda_{s}}\left(\frac{1}{D_{A}^{2}} \int_{0}^{\lambda^{\prime}} Q_{L} D_{A}^{2} M d \lambda^{\prime \prime}\right) d \lambda^{\prime}$.

Since we are only considering first order effects of $Q_{L}$ one can use iterations to solve this integro-differential equations in orders of the curvature deviation. One can see that the leading order is

$M=\mathbb{I}-\int_{0}^{\lambda_{s}}\left(\frac{1}{D_{A}^{2}} \int_{0}^{\lambda^{\prime}} Q_{L} D_{A}^{2} d \lambda^{\prime \prime}\right) d \lambda^{\prime}$.

This is the main equation which describes the distortions at first order of isolated distributions over the cosmological background here considered. 


\subsection{Optical scalars}

It was mentioned before that the matrix $\mathcal{A}$ containing the optical scalars is given by:

$\mathcal{A}=\left(1-\kappa_{c}\right) M$.

It will be used together with equation (159) to obtain the optical scalars. This task is most easily done working with the real and imaginary parts of the deviation vector $\varsigma$; namely $\varsigma=\varsigma_{R}+i \varsigma_{I}$ and the real and imaginary parts of the Weyl curvature scalar, $\Psi_{0}^{L}=\Psi_{0 R}^{L}+i \Psi_{0 I}^{L}$.

One finds the following structure in the optical matrix

$\mathcal{A}=\left(1-\kappa_{c}\right)\left(\begin{array}{cc}1-\kappa_{L}-\gamma_{1 L} & -\gamma_{2 L} \\ -\gamma_{2 L} & 1-\kappa_{L}+\gamma_{1 L}\end{array}\right)$

where the intrinsic optical terms to the additional lens $\left(\kappa_{L}, \gamma_{1 L}, \gamma_{2 L}\right)$ are explicitly

$\kappa_{L}=\int_{0}^{\lambda_{s}}\left(\frac{1}{D_{A}^{2}} \int_{0}^{\lambda^{\prime}} \Phi_{00}^{L} D_{A}^{2} d \lambda^{\prime \prime}\right) d \lambda^{\prime}$,

$\gamma_{1 L}=\int_{0}^{\lambda_{s}}\left(\frac{1}{D_{A}^{2}} \int_{0}^{\lambda^{\prime}} \Psi_{0 R}^{L} D_{A}^{2} d \lambda^{\prime \prime}\right) d \lambda^{\prime}$,

$\gamma_{2 L}=\int_{0}^{\lambda_{s}}\left(\frac{1}{D_{A}^{2}} \int_{0}^{\lambda^{\prime}} \Psi_{0 I}^{L} D_{A}^{2} d \lambda^{\prime \prime}\right) d \lambda^{\prime} ;$

where the matrix in (160) refers to the physical frame in the image plane.

The subindex $L$ is introduced at this moment to distinguish quantities depending on the isolated inhomogeneities in contrast to background quantities such as $\kappa_{c}$.

Then, one can write the optical scalars for the whole system of an additional lens regarded over a $\mathrm{R}-\mathrm{W}$ spacetime:

$$
\begin{aligned}
\kappa & =\left(1-\kappa_{c}\right) \kappa_{L}+\kappa_{c}, \\
\gamma_{1} & =\left(1-\kappa_{c}\right) \gamma_{1 L}, \\
\gamma_{2} & =\left(1-\kappa_{c}\right) \gamma_{2 L} .
\end{aligned}
$$

One can see that the contribution of the background is two fold; on one hand, it is present in the intrinsic optical scalar trough the geometric distance and the area distance and, on the other hand it also appears by means of the modulating factor $\left(1-\kappa_{c}\right)$.

The above expressions are the generalization for the optical scalars of a lens in the standard cosmological context.

In the simpler case of a Minkowski or a Milne background spacetime, where $D_{A}=\lambda$, one arrives at the expression already presented in Gallo \& Moreschi (2011); they are:

$$
\begin{aligned}
\kappa & =\frac{1}{\lambda_{s}} \int_{0}^{\lambda_{s}} \lambda\left(\lambda_{s}-\lambda\right) \Phi_{00}^{L} d \lambda^{\prime}, \\
\gamma_{1} & =\frac{1}{\lambda_{s}} \int_{0}^{\lambda_{s}} \lambda\left(\lambda_{s}-\lambda\right) \Psi_{0 R}^{L} d \lambda^{\prime}, \\
\gamma_{2} & =\frac{1}{\lambda_{s}} \int_{0}^{\lambda_{s}} \lambda\left(\lambda_{s}-\lambda\right) \Psi_{0 I}^{L} d \lambda^{\prime} ;
\end{aligned}
$$

in which the double integral has been expressed into a single one by means of a integration by parts.

\section{THE AXIALLY SYMMETRIC CASE}

The expressions presented in the last sections do not involve any assumption on the nature and geometry of the source; in this section, instead, we discuss models with axial symmetry, in which the axis of symmetry lies along the line of sight passing through the central region of the distribution. We are interested then in the study of a photon traveling along a direction parallel to the axis of symmetry which has an impact parameter $J$ with respect to the centre of the distribution. For this purpose we will take an adapted coordinate system to the situation in which two mutually orthogonal directions $x$ and $z$ are considered. They are also orthogonal to the direction $y$ in which the photons travel. Due to the symmetry of the lens it will be also useful to work in terms of the angle $\vartheta$ between the $z$-axis and the path of the photon.

Then, with this setting one notes that the component $\Psi_{0}^{L}$ is a spin zero real quantity, and it depends on the $(J, \lambda)$ coordinates, while the component $\Psi_{0}^{L}$ is a spin two complex quantity and it has the functional dependence

$\Psi_{0}^{L}=\left|\Psi_{0}^{L}\right| e^{2 i \vartheta+\text { phase }} ;$

where the phase is gauge dependent.

As it was mentioned in Gallo \& Moreschi (2011), it is usual to define the real quantity $\psi_{0}^{L}(J, \lambda)$ associated to the Weyl contribution from

$\Psi_{0}^{L}(J, \lambda, \vartheta)=-\psi_{0}^{L}(J, \lambda) e^{2 i \vartheta}$.

With this definition one writes for the intrinsic optical scalars of the lens:

$$
\begin{aligned}
\kappa_{L}(J) & =\int_{0}^{\lambda_{s}}\left(\frac{1}{D_{A}^{2}} \int_{0}^{\lambda^{\prime}} \Phi_{00}^{L}(J, \lambda) D_{A}^{2} d \lambda^{\prime \prime}\right) d \lambda^{\prime} \\
\gamma_{c L}(J, \vartheta) & =-e^{2 i \vartheta} \int_{0}^{\lambda_{s}}\left(\frac{1}{D_{A}^{2}} \int_{0}^{\lambda^{\prime}} \psi_{0}^{L}(J, \lambda) D_{A}^{2} d \lambda^{\prime \prime}\right) d \lambda^{\prime} ;
\end{aligned}
$$

where the complex shear is normally expressed in terms of its real and imaginary parts, namely $\gamma_{c L} \equiv \gamma_{1 L}+i \gamma_{2 L}$.

The complex form for the shear components invite us to define the real quantity $\gamma_{L}(J)$ as

$\left(\gamma_{1 L}+i \gamma_{2 L}\right)(J, \vartheta) \equiv-\gamma_{L}(J) e^{2 i \vartheta} ;$

so that one simply has

$\gamma_{L}(J)=\int_{0}^{\lambda_{s}}\left(\frac{1}{D_{A}^{2}} \int_{0}^{\lambda^{\prime}} \psi_{0}^{L}(J) D_{A}^{2} d \lambda^{\prime \prime}\right) d \lambda^{\prime}$.

\section{THE THIN LENS APPROXIMATION}

The common situation in the cosmological scenario is the one in which the typical size $\delta_{l}$ of the lens is much smaller than the distance from the observer to the lens $\lambda_{l}$, and than the distance from the lens to the source $\lambda_{l s}$. This configuration is referred to as a thin lens.

Following the same lines presented in article Gallo \& Moreschi (2011) we will assume that the scalars of curvature $\left\{\Phi_{00}^{L}, \Psi_{0}^{L}\right\}$, denoted generically as $\bar{C}^{L}$ will be sharply peaked around $\lambda_{l}$, where the lens is located. This implies that the following approximation must to hold

$\bar{C}^{L}(\lambda) \equiv \int_{0}^{\lambda} \delta C\left(\lambda^{\prime}\right) d \lambda^{\prime} \cong\left\{\begin{array}{ll}0, & \forall \lambda<\lambda_{l}-\delta_{l} \\ \widehat{C}^{L}, & \forall \lambda \geq \lambda_{l}+\delta_{l}\end{array} ;\right.$

where $\delta_{l} \ll \lambda_{l}, \delta_{l} \ll \lambda_{s}$ and $\delta_{l} \ll \lambda_{l s}\left(\lambda_{l s}=\lambda_{s}-\lambda_{l}\right)$. 
We make use of this assumption to simplify the integrand inside of parenthesis in the expressions in equations $(161,162,163)$. Let us note that

$$
\begin{aligned}
\frac{1}{D_{A}^{2}} \int_{0}^{\lambda} C^{L} D_{A}^{2} d \lambda^{\prime} & =\widehat{C}^{L}-\frac{1}{D_{A}^{2}} \int_{0}^{\lambda} \frac{d D_{A}^{2}}{d \lambda^{\prime}} \bar{C}^{L}\left(\lambda^{\prime}\right) d \lambda^{\prime} \\
& =\widehat{C}^{L}-\frac{\widehat{C}^{L}}{D_{A}^{2}}\left(D_{A}^{2}-D_{A}^{2}\left(\lambda_{l}\right)\right) \\
& =\widehat{C}^{L} \frac{D_{A}^{2}\left(\lambda_{l}\right)}{D_{A}^{2}}
\end{aligned}
$$

where it is clear that one must have $\lambda_{l} \leqslant \lambda$. Then, one can take another integral to arrive at reduced expressions for the optical scalars; one finds

$$
\int_{0}^{\lambda_{s}}\left(\frac{1}{D_{A}^{2}\left(\lambda^{\prime}\right)} \int_{0}^{\lambda^{\prime}} C^{L} D_{A}^{2} d \lambda^{\prime \prime}\right) d \lambda^{\prime}=\widehat{C}^{L} D_{A}^{2}\left(\lambda_{l}\right) \int_{\lambda_{l}}^{\lambda_{s}} \frac{d \lambda^{\prime}}{D_{A}^{2}}
$$

where we have neglected terms of order $\mathscr{O}\left(\frac{\delta_{l}}{\lambda_{l}}\right)$.

Since the factor in the last equation will appear recurrently we define, for the sake of simplicity in the notation, the symbol:

$\mathbf{D}_{l s} \equiv D_{A}^{2}\left(\lambda_{l}\right) \int_{\lambda_{l}}^{\lambda_{s}} \frac{d \lambda^{\prime}}{D_{A}^{2}\left(\lambda^{\prime}\right)}$

Then, the intrinsic optical scalars of the lens acquire a simpler form:

$$
\begin{aligned}
\kappa_{L} & =\mathbf{D}_{l s} \widehat{\Phi}_{00}^{L}, \\
\gamma_{1 L} & =\mathbf{D}_{l s} \widehat{\Psi}_{0 R}^{L}, \\
\gamma_{2 L} & =\mathbf{D}_{l s} \widehat{\Psi}_{0 I}^{L} ;
\end{aligned}
$$

while the optical scalars of the whole system

$$
\begin{aligned}
\kappa & =\left(1-\kappa_{c}\right) \mathbf{D}_{l s} \widehat{\Phi}_{00}^{L}+\kappa_{c}, \\
\gamma_{1} & =\left(1-\kappa_{c}\right) \mathbf{D}_{l s} \widehat{\Psi}_{0 R}^{L}, \\
\gamma_{2} & =\left(1-\kappa_{c}\right) \mathbf{D}_{l s} \widehat{\Psi}_{0 I}^{L} ;
\end{aligned}
$$

where, from the above notation one is using:

$\widehat{\Phi}_{00}^{L}=\int_{0}^{\lambda_{s}} \Phi_{00}^{L} d \lambda^{\prime}$,

and

$\widehat{\Psi}_{0}^{L}=\int_{0}^{\lambda_{s}} \Psi_{0}^{L} d \lambda^{\prime}$

Notice that since $\kappa_{c}$ is order $\mathscr{O}\left(Q_{B}\right)$, and $\kappa_{L}$ and $\gamma_{L}$ are order $\mathscr{O}\left(Q_{L}\right)$; one finds terms of order order $\mathscr{O}\left(Q_{B} Q_{L}\right)$ in equations (182)-(184).

In section 10.1 we will show the connection of the above expressions with usual formulae present in the literature. For the moment, let us note that, in the limit of no cosmological background one has that $D_{A}$ converges to $\lambda$ and

$\mathbf{D}_{l s} \rightarrow \frac{\lambda_{l} \lambda_{l s}}{\lambda_{s}}$

so that in this way one arrives at the usual expressions found in the studies of gravitational lenses over a flat backgroundGallo \& Moreschi (2011).

It is worthwhile to remark that the expressions (179)(181) are built out of two distinctive factors; namely the $\mathbf{D}_{l s}$ and the hated quantities that are calculated from the curvature of the lens. The first factor does not depend on the possible motion of the lens, and it only depends on the cosmological scenario. Instead the hated quantities depend on the possible motion of the lens; which is unavoidable in the cosmological context. In the next two subsection we concentrate on both factors.

\subsection{Alternative expression for the factor $\mathbf{D}_{l s}$}

The term, $\mathbf{D}_{l s}$, which only contains information about the cosmology, can be written in a more clarifying form using the coordinates $(u, \chi)$ discussed in section 3.1.2. In fact, we show that the integrand appearing in equation (178) is a total derivative along the null geodesic.

Let us note that from equation (60) and the fact that $\frac{\partial}{\partial \lambda}=-\ell$ one has the following relation

$d \lambda=\frac{A^{2}(u, \chi)}{A\left(t_{0}\right)} d \chi$

which means that

$$
\begin{aligned}
\mathbf{D}_{l s} & =\frac{D_{A}^{2}\left(\chi_{l}\right)}{A\left(t_{0}\right)} \int_{\chi_{l}}^{\chi_{s}} \frac{d \chi}{f_{k}^{2}(\chi)} \\
& =\frac{D_{A}^{2}\left(\chi_{l}\right)}{A\left(t_{0}\right)} \frac{f_{k}\left(\chi_{s}-\chi_{l}\right)}{f_{k}\left(\chi_{s}\right) f_{k}\left(\chi_{l}\right)} \\
& =\frac{1}{\left(1+z_{l}\right)} \frac{D_{A}\left(\chi_{s}-\chi_{l}\right) D_{A}\left(\chi_{l}\right)}{D_{A}\left(\chi_{s}\right)}
\end{aligned}
$$

where it must be understood that $z_{l}$ is the redshift at the place of the lens and $D_{A}\left(\chi_{s}-\chi_{l}\right)$ is the angular diameter distance measured by a fundamental observer at the coordinates $\left(u, \chi_{l}\right)$

$D_{A}\left(\chi_{s}-\chi_{l}\right)=A\left(u, \chi_{s}\right) f_{k}\left(\chi_{s}-\chi_{l}\right)$.

In what follows we will use a short notation in which we only retain the subindex of the coordinate $\chi$, namely:

$\mathbf{D}_{l s}=\frac{1}{1+z_{l}} \frac{D_{A_{l s}} D_{A_{l}}}{D_{A_{s}}}$.

Let us observe that the presence of the redshift factor $1+z_{l}$ could be source of confusion if one consider the case of a Milne (vacuum) spacetime where $D_{A}=\lambda$ but $z_{l} \neq 0$ since in this case it appear that we will not recover the expression (187). This is just apparent since in this case one has that equation (190) becomes $\left(1+z_{l}\right)\left(\lambda_{s}-\lambda_{l}\right)$ as one can easily check. This is due to the fact that the area distance depends on the motion of the observer which in this case correspond to the comoving cosmic observer. In other words, one has for Milne spacetime

$D_{A_{l s}} \rightarrow \bar{\lambda}_{s}-\bar{\lambda}_{s}=\left(1+z_{l}\right)\left(\lambda_{s}-\lambda_{l}\right)$;

where $\bar{\lambda}$ refers to the affine parameter defined by equation (10) respect to the worldline of the comoving cosmic observer.

\subsection{Integration on the curvature for moving lenses}

In principle, expressions (185) and (186) have all the information one needs to complete the calculation of the optical scalars. But in the case the lens is moving one might like to refer the calculation to its intrinsic rest frame. To do this 
one can think in leaving the null frame of the observer fixed an calculate the curvature of moving sources, or one can think in leaving the geometry unaffected and only change the frame of observation by the appropriate boost. From the GHP formalismGeroch et al. (1973) one knows that both integrands have boost weight 2 . Then, taking into account the change in the affine parameter for the boosted frame, one deduces that for a local stationary piece of of spacetime which is moving relative to the observer with four velocity $v^{a}$, the expressions (185) and (186) are related with respect to the non-moving case by a factor

$\frac{1}{\ell \cdot v}=1+z_{v}$.

Let us emphasize that $z_{v}$ does not need to agree with $z_{l}$.

Then, one can see that expressions (179)-(181), have all the same structure; namely, if $\mathrm{o}_{L v}$ is the optical scalar for a lens with velocity $v$, one can express

$$
\begin{aligned}
\mathrm{o}_{L v} & =\mathbf{D}_{l s} \widehat{C}_{v}^{L} \\
& =\mathbf{D}_{l s}\left(1+z_{v}\right) \widehat{C}^{L} \\
& =\frac{\left(1+z_{v}\right)}{\left(1+z_{l}\right)} \frac{D_{A_{l s}} D_{A_{l}}}{D_{A_{s}}} \widehat{C}^{L} \\
& =\frac{\left(1+z_{v}\right)}{\left(1+z_{l}\right)} \mathrm{o}_{L} ;
\end{aligned}
$$

where we are using the notation $\widehat{C}_{v}^{L}$ for the moving lens and $\widehat{C}^{L}$ for the lens at rest with respect to the observer.

The simplicity of equation (194), and in its derivation, should not hide that this is a noteworthy expression that synthesizes all that one needs to take into account in the motion of the lenses; therefore in this way we simplify previous worksKopeikin \& Schaefer (1999); Frittelli (2003); Wucknitz \& Sperhake (2004) on the subject of moving gravitational lenses, and generalized its application to the cosmological scenario.

\section{A note regarding $\Sigma_{c r}$ :}

At this point we would also like to take the opportunity to make a remark regarding the notion of critical mass density used in several previous works and textbooks.

We have noticed before that the contribution to the optical gravitational lens scalars appearing in (179)-(181) are composed of two distinctive factors involving direct contributions from the cosmological background and the lens respectively. When comparing with the textbookSchneider et al. $(1992,2006)$ recipe based on the $\Sigma / \Sigma_{c r}$ approach, we should relate our $\mathbf{D}_{l s}$ with $1 / \Sigma_{c r}$ and $\widehat{C}_{v}^{L}$ with $\Sigma$. Let us point out that in a strict sense, we have found that $\mathbf{D}_{l s}$ does not coincides with $1 / \Sigma_{c r}$ as defined bySchneider et al. (2006)

$$
\frac{1}{\Sigma_{c r}} \equiv \frac{4 \pi G}{c^{2}} \frac{D_{A}\left(\chi_{s}-\chi_{l}\right) D_{A}\left(\chi_{l}\right)}{D_{A}\left(\chi_{s}\right)}
$$

since, up to the numerical factor with the physical constants, we note that the factor involving the redshift of the lens is missing. This is important because in reference Schneider et al. (2006) it has been claimed that to do calculations in the cosmological context, one 'only' needs to replace the flat area distances with the cosmological ones.

\section{A note regarding $\Sigma$ :}

Furthermore, in the textbook Schneider et al. (1992), near their equations (4.19) and (4.20), it is calculated that the deflecting angle is unaffected, by first order effects on the velocity of the lens. Then since the quantity $\Sigma$ used in these references is just linear in the deflecting angle, one would deduce that $\Sigma$ is unchanged by the motion of the lens. We have shown above that this is not correct.

This means that it is wrong to generalize the common expressions appearing in weak lensing studies in the case of a flat background to the cosmological scenario, just by changing distances to angular diameter distances. We will return to this point below when presenting basic examples.

\section{STATIONARY SPHERICALLY SYMMETRIC MOVING THIN LENSES}

Let us consider the case in which locally, the gravitational lens, is represented by a stationary spherically symmetric line element. Then, in a neighbourhood of the lens, we can set a coordinate system where the line element of the geometry can be expressed as:

$d s_{L}^{2}=e^{2 \Phi(\mathbf{r})} d \mathbf{t}^{2}-\frac{d \mathbf{r}^{2}}{1-\frac{2 M(\mathbf{r})}{\mathbf{r}}}-\mathbf{r}^{2}\left(d \theta^{2}+\sin ^{2}(\theta) d \phi^{2}\right) ;$

which is completely determined by the functions $M(\mathbf{r})$ and $\Phi(\mathbf{r})$.

It is important to emphasize that the thin lens approximation as given in expressions (179)-(181) can be applied to this situation. When this is done one notes also that the information provided by the underlined cosmology will appear only trough the factor (191) containing the angular diameter distances and the redshift.

Furthermore, comparing the above equations (179)(181) with equations (78)-(80) of reference Gallo \& Moreschi (2011), and realizing that in the thin lens approximation, what matters is the impact parameter $J$ from the center; one can note that all the discussion of section $\mathrm{V}$ of Gallo \& Moreschi (2011) can be applied also to the cosmological scenario; taking into account the motion of the lens discuss previously. This means that we can express the optical scalars in terms of the energy-momentum components and the function $M(\mathbf{r})$ as:

$$
\begin{aligned}
\kappa_{L}(J)= & \frac{4 \pi G}{c^{2}} \mathbf{D}_{l s}\left(1+z_{v}\right) \int_{-\infty}^{\infty}\left[\varrho(\mathbf{r})+\frac{P_{r}(\mathbf{r})}{c^{2}}\right. \\
& \left.+\frac{J^{2}}{c^{2} \mathbf{r}^{2}}\left(P_{t}(\mathbf{r})-P_{r}(\mathbf{r})\right)\right] d y
\end{aligned}
$$

and

$$
\begin{aligned}
\gamma_{L}(J)= & \frac{G}{c^{2}} \mathbf{D}_{l s}\left(1+z_{v}\right) \int_{-\infty}^{\infty} \frac{J^{2}}{\mathbf{r}^{2}}\left[\frac{3 M(\mathbf{r})}{\mathbf{r}^{3}}\right. \\
& \left.-\left(\varrho(\mathbf{r})+\frac{P_{t}(\mathbf{r})}{c^{2}}-\frac{P_{r}(\mathbf{r})}{c^{2}}\right)\right] d y
\end{aligned}
$$

where $\varrho$ denotes the energy density of the distribution and $P_{r}(r)$ and $P_{t}(r)$ are the spacelike components of the energymomentum tensor. In order to simplify the notation we are omitting the upper indices ' $\mathrm{L}$ ' in the curvature expressions. The integration variable $y$ is defined as in Gallo \& Moreschi (2011):

$\mathbf{r}^{2} \equiv J^{2}+y^{2}$ 
It is probably worthwhile noting that the standard assumption in cosmological studies is to neglect the spacelike components of the energy-momentum tensor.

Equations (197) and (198) are very useful expressions which allow us to write in a simple manner a relation between the optical scalars of the gravitational lens and the matter content; and constitute the generalization of the pair of equations (183) of Gallo \& Moreschi (2011) to the cosmological context.

In the static spherically symmetric lens discussed above, we have assumed that locally the geometry has this behavior so that we can use the standard line element, where the curvature need not be small with respect to the cosmological background. However one could also deal with the situation in which one has a local static spherically symmetric distribution of matter which is small with respect to the local cosmic energy density. In this situation, we can still use the above expressions, now with the understanding that the quantities refer with respect to the cosmological background. So that, for example, in the discussion of the energy density, if we denote with $\varrho_{c}$ the cosmological energy density corresponding to the position of the lens, one would use $\varrho^{L}$ instead of $\rho$ in the above expressions, and would have $\varrho^{L}=\varrho_{\text {proper }}-\varrho_{c}$.

\subsection{Examples}

In this subsection we apply the generalized expressions for the optical scalars of a monopole mass, for the well known isothermal mass density distribution, and we also present a peculiar geometry of lens. We take the opportunity to point out an interesting relation that this unusual distribution shares with the isothermal one.

\subsubsection{A monopole mass (Schwarzschild)}

Let us consider here a monopole mass characterized by a mass $M=$ constant; which it could be moving with velocity $v$ with respect to the observer. For a such lens one finds that

$\kappa_{L}=0$,

$\gamma_{L}=\frac{4 G}{c^{2}} \mathbf{D}_{l s}\left(1+z_{v}\right) \frac{M}{J^{2}}$

then the total convergence and shear are:

$\kappa=\kappa_{c}$,

$$
\begin{aligned}
\gamma & =\left(1-\kappa_{c}\right) \frac{4 G}{c^{2}} \mathbf{D}_{l s}\left(1+z_{v}\right) \frac{M}{J^{2}} \\
& =\frac{4 G}{c^{2}} \frac{\left(1-\kappa_{c}\right)}{\left(1+z_{l}\right)} \frac{D_{A_{s l}} D_{A_{l}}}{D_{A_{s}}}\left(1+z_{v}\right) \frac{M}{J^{2}}
\end{aligned}
$$

\subsubsection{The isothermal profile}

The isothermal profile is characterized by a mass density function of the form

$$
\varrho(\mathbf{r})=\frac{\sigma^{2}}{2 G \pi \mathbf{r}^{2}} \text {. }
$$

This distribution appears in many astrophysical studies in which it is often used as profile for stellar dynamics models and for the study of the differential rotation of galaxies whit dark matter among other applications. The parameter $v \equiv$ $\sqrt{2} \sigma$ has unit of velocity and it is assumed to satisfy $\mathrm{v} \ll c$; which implies that pressures in this model can be neglected. This characteristic velocity of the isothermal profile should not be confused with the possible velocity of the lens $v$. Then, the matter content is characterized by

$M(\mathbf{r})=\frac{2 \sigma^{2}}{G} \mathbf{r}$,

$P_{r}(\mathbf{r})=P_{t}(\mathbf{r}) \approx 0$

together with equation (204).

It yields the following intrinsic optical scalar for the lens:

$\kappa_{L}=\frac{\mathbf{D}_{l s}}{c^{2}}\left(1+z_{v}\right) \frac{2 \pi \sigma^{2}}{J}$,

$\gamma_{L}=\frac{\mathbf{D}_{l s}}{c^{2}}\left(1+z_{v}\right) \frac{2 \pi \sigma^{2}}{J} ;$

and if we include the proper contribution from the background we obtain:

$$
\begin{aligned}
\kappa & =\left(1-\kappa_{c}\right) \frac{\mathbf{D}_{l s}}{c^{2}}\left(1+z_{v}\right) \frac{2 \pi \sigma^{2}}{J}+\kappa_{c} \\
& =\frac{\left(1-\kappa_{c}\right)}{\left(1+z_{l}\right)} \frac{D_{A_{s l}} D_{A_{l}}}{c^{2} D_{A_{s}}}\left(1+z_{v}\right) \frac{2 \pi \sigma^{2}}{J}+\kappa_{c} \\
\gamma & =\left(1-\kappa_{c}\right) \frac{\mathbf{D}_{l s}}{c^{2}}\left(1+z_{v}\right) \frac{2 \pi \sigma^{2}}{J} \\
& =\frac{\left(1-\kappa_{c}\right)}{\left(1+z_{l}\right)} \frac{D_{A_{s l}} D_{A_{l}}}{c^{2} D_{A_{s}}}\left(1+z_{v}\right) \frac{2 \pi \sigma^{2}}{J}
\end{aligned}
$$

\subsubsection{Peculiar anisotropic solution}

The last example is an exact solution of the Einstein equation which describes very well the phenomenology of the dark matter in astrophysical systems which was presented in Gallo \& Moreschi (2012). It posses a non-conventional energy-momentum tensor whose components are:

$$
\begin{aligned}
\varrho(\mathbf{r}) & =0, \\
P_{r}(\mathbf{r}) & =\frac{c^{4}}{4 \pi G \mathbf{r}^{2} \ln \left(\frac{\mathbf{r}}{\mu}\right)}, \\
P_{t}(\mathbf{r}) & =0
\end{aligned}
$$

with $\mu$ a constant of the distribution. The solution satisfies the weak and strong energy conditions and it was shown that the total mass of the distribution $M(\mathbf{r})$ vanishes.

In different application of this geometry to problems involving dark matter it was found that in the range of interest $\ln \mathbf{r}$ was much smaller than $-\ln \mu$. This means that for any practical purposes, the $\log$ factor could be identified with a constant; in other words one could take $4 \Delta^{2} \cong \frac{c^{2}}{\ln \left(\frac{r}{\mu}\right)}$. Then, by using equations (197) and (198) one arrives at:

$\begin{aligned} \kappa_{L}(J) & =\frac{\mathbf{D}_{l s}}{c^{2}}\left(1+z_{v}\right) \frac{2 \pi \Delta^{2}}{J}, \\ \gamma_{L}(J) & =\frac{\mathbf{D}_{l s}}{c^{2}}\left(1+z_{v}\right) \frac{2 \pi \Delta^{2}}{J} .\end{aligned}$

Therefore we see that this type of models behave as an isothermal profile with velocity dispersion $v=\sqrt{2} \Delta$ when it 
is regarded as a gravitational lens. The shear and the convergence are

$$
\begin{aligned}
\kappa & =\left(1-\kappa_{c}\right) \frac{\mathbf{D}_{l s}}{c^{2}}\left(1+z_{v}\right) \frac{2 \pi \Delta^{2}}{J}+\kappa_{c} \\
& =\frac{\left(1-\kappa_{c}\right)}{\left(1+z_{l}\right)} \frac{D_{A_{s l}} D_{A_{l}}}{c^{2} D_{A_{s}}}\left(1+z_{v}\right) \frac{2 \pi \Delta^{2}}{J}+\kappa_{c} \\
\gamma & =\left(1-\kappa_{c}\right) \frac{\mathbf{D}_{l s}}{c^{2}}\left(1+z_{v}\right) \frac{2 \pi \Delta^{2}}{J} \\
& =\frac{\left(1-\kappa_{c}\right)}{\left(1+z_{l}\right)} \frac{D_{A_{s l}} D_{A_{l}}}{c^{2} D_{A_{s}}}\left(1+z_{v}\right) \frac{2 \pi \Delta^{2}}{J} .
\end{aligned}
$$

It is probably valuable to remark that if one were to use the textbook recipe, based on the $\Sigma / \Sigma_{\text {cr }}$ quantities, one would obtain zero for all the optical scalars in this case, since $\Sigma=0$.

\section{A note on the examples:}

These examples give the opportunity to show the main differences of our general expressions with the paradigm based on the $\Sigma / \Sigma_{\text {cr }}$ approach. The first two examples show that our expressions include the cosmic contribution by the factor involving the cosmic convergence gravitational lens scalar $\kappa_{c}$ and the contribution of the general velocity of the lens by the factor involving the redshift $z_{v}$. For lenses which are comoving with the fundamental observers of the background R-W frame, one has a cancellation of this factor with the denominator involving the cosmic background redshift; but the contribution from the the cosmic convergence gravitational lens scalar remains, so that even in this case, our results do no agree with the simplified model based on the $\Sigma / \Sigma_{\text {cr }}$ recipe.

In standard presentations of the above mentioned paradigm the issue of possible motion of the lens is completely neglected, so that they omit the two effects that produces the two factors shown above; and therefore their cancellation, for the case of a comoving lens, has gone unnoticed in previous works.

Notably the last example demonstrate the inability of the mentioned paradigm to deal with geometries that have been shown to be useful for the description of the dark matter phenomena.

\section{FINAL COMMENTS}

In this work we have presented a detailed systematic study of gravitational lens optical scalars in the cosmological context. We have also included new expressions for them, which allow for a general energy-momentum content of the lens and at the same time they contain the corrections due to the motion of the lens. We also have presented new formulae for the relation of the observed magnitudes with the optical scalars through the intensity magnifications we define here.

In our approach we follow the usual framework for the study of gravitational lens effects, in which one compares the lensed situation with the unlensed one; in which no curvature is present. In order to take into account the subtleties appearing in the discussion of lenses over a curved spacetimes, such as R-W, it was necessary to review the basic language and interpretation of observations; which has been done in section 2. In particular we have remarked that the natural and universal notion of distance, that can be applied to any spacetime, is the affine distance $\lambda$.
It is interesting to note that the whole $\mathrm{R}-\mathrm{W}$ geometry can be characterized as a gravitational lens in its own right; which is thick, only producing convergence but not shear. It is also worthwhile to remark that our expressions for the cosmic convergence are valid for arbitrary large observing angles.

We have studied also the existence of an additional lens in a R-W scenario, and have presented the new general expressions for the optical scalars, namely equations (161)(166). One should note that in several works sometimes the effects of additional lenses are presented only with respect to a R-W background, which corresponds only to equations (161)-(163); but in this case one would miss some effects of cosmological origin, that we include here explicitly.

Although there are excellent textbooksSchneider et al. $(1992,2006)$ in the literature that present a complete view of the subject of gravitational lens in a clear pedagogical way, they rely on simplifying assumptions that severely limit their use in detailed research of general astrophysical situations. In particular they assume a typical lens is of Newtonian nature and not moving. So, our new expressions possess the interesting feature that they are not restricted to Newtonian like distributions of massive scatterer, nor are confined to be at rest; instead, they allow for a very general class of energymomentum tensors which make these formulas a useful tool to address some of the difficulties related to the missing mass problem in the Universe. In this way we generalize expressions appearing in textbooksSchneider et al. (1992, 2006), reviewsWambsganss (1998); Bartelmann \& Schneider (2001); Bartelmann (2010) and premises of research articlesKling \& Frittelli (2008); Liao et al. (2016); Holanda et al. (2016).

It is worthwhile to remark that our expressions for the gravitational lens optical scalar are presented in terms of curvature components; that is, prior to the choice of the field equation one would like to use. But in all the examples we have discussed, we have used the Hilbert-Einstein field equations.

The thin lens approximation has provenFrittelli \& Kling (2011) to be a good working hypothesis for a variety of systems. Surprisingly, when the thin lens approximation is considered we have noticed the appearance of a factor involving the redshift of the lens; as is indicated in equation (189); which has not been considered previously. This means that the straightforward change of angular diameter distance, used in the case of flat background, to the angular diameter distance used on a R-W background, is not enough to obtain the complete expression. We remark this because several authors have suggested this wrong technique, as for example in reference Schneider et al. (2006); where the factor involving the redshift is missing. In order to remark the conceptual difference of our approach to those found in standard textbooks, we have presented the general situation in which the lenses might be moving with arbitrary velocity. This in turn includes another redshift factor associated to the motion of the lens which of course need not coincide with the velocity of the assumed fundamental cosmic observers of a R-W background.

These equations are additionally useful and relevant to works concerning test of fundamental geometric relations in observational cosmology; which are based on the use of gravitational lensingHolanda et al. (2016); Liao et al. (2016).

We have considered few classic examples of static and 
spherically symmetric lenses, and applied the equations for the gravitational lens optical scalars. This is easily done, since we have provided with expressions that relate the optical scalars to the matter content of the lens.

It is probably worthwhile to point out that in our presentation we have not used the notion of bending angle at all; since it is not essential, but it is useful to show the difference with the standard approach based on this concept. For those that would like to read a discussion of both concepts for the case of gravitational lens with symmetries, we refer to our previous article Gallo \& Moreschi (2011). In relation to this, let us also mention that in the $\Sigma / \Sigma_{\text {cr }}$ paradigm, which is based in the bending angle concept, one can not discuss the cosmic convergence $\kappa_{c}$ appearing in R-W spacetimes; since there is no bending of the light rays in this case (See also Pyne \& Birkinshaw (1996)). For this reason within this paradigm there appear no discussions of the observations of supernovae; instead we have shown here that the luminosity observations of a supernova can be expressed in the terms of the gravitational lens optical scalar of the cosmological spacetime.

The connection with the observed magnitudes is done with our introduction of the intensity magnification $\tilde{\mu}$, defined in terms of the fluxes, and we have also provided with an expression of the distance modulus $m-M$, in terms of it, given in equations (98) and (99). In order to make contact with the way in which the community describes the behavior of the distance modulus, we have also introduced an astrophysical cosmic intensity magnification $\mu_{c}^{\prime}$ that is thought as a function of redshift. One can also express the distance modulus in terms of this magnification as we have done in (100). It is important to remark that the physical intensity magnification $\tilde{\mu}$ turns out to depend only on the affine distance $\lambda$; and that it coincides with the gravitational lens magnification $\mu$. Therefore the distance modulus as expressed in equation (99), valid in a general spacetime, only depends on the gravitational lens scalars and kinematical data. Instead, when expressing the distance modulus in terms of the astrophysical cosmic intensity magnification $\mu_{c}^{\prime}$, it requires the assumption of a cosmological model, so that a distance-redshift relations can be applied. It is for this reason that something very peculiar happened with the way in which the data of supernovae has been studied, namely: one can see from equations (99) and (23) that, since in the calculation of the gravitational lens optical scalars only intervene the traceless part of the Ricci tensor and the Weyl tensor, the observations of the supernovae fluxes do not depend on a possible cosmological constant, which only affects the trace of the Ricci tensor. However in celebrated works, as Riess et al. (1998); Perlmutter et al. (1999), the authors have studied the relation between the behavior of the supernovae fluxes with redshift, by the use of equation (100) with the assumption of a cosmological model, and argued that a cosmological constant explains the observations. The peculiarity is also related to this: from tables 1 and 2 one can see that the physical intensity magnification $\tilde{\mu}$ is always greater than one; so that if one applies (99) to describe the observed luminosity supernovae, one would find that they are brighter than in the flat case; instead, in the analysis of Riess et al. (1998); Perlmutter et al. (1999) the claim is that since the observed luminosity of supernovae is fainter
(See table 1) than expected as a function of redshift, then a cosmological constant would account for it.

It should be noticed that our arguments leading to the new expression (99), should not be confused with the standard arguments involving the statements of constant surface brightness as appear in textbooksSchneider et al. (1992). Our argument relies on the validity of the Etherington theorem, which is applicable to general spacetimes and classes of lenses.

Let us note that in our direct approach to the study of gravitational lens scalars, the discussion of moving lenses is straight forward and it can be done in few lines, as expressed in the deduction of equation (194). Instead in previous approaches based on the bending angle concept, the discussions require much more complicated algebraKopeikin \& Schaefer (1999); Frittelli (2003); Wucknitz \& Sperhake (2004).

Within the approximation of thin lenses we have considered configurations with a single lens but the treatment can easily be extended to complex arrangements of several lenses. That is, one could consider having $N$ lenses, placed at cosmological distances $\lambda_{1}, \ldots, \lambda_{N}$, and apply the techniques explained above. We will tackle this problem in a further work.

\section{ACKNOWLEDGEMENTS}

We have benefited from comments from an anonymous Reviever. We acknowledge support from CONICET, SeCyTUNC and Foncyt.

\section{REFERENCES}

Ade P. A. R., et al., 2014, Astron. Astrophys., 571, A16

Baker J. G., Boggs W. D., Centrella J., Kelly B. J., McWilliams

S. T., Miller M. C., van Meter J. R., 2008, Astrophys. J., 682

Bardeen J. M., 1980, Phys. Rev., D22, 1882

Bartelmann M., 2010, Class. Quantum Grav., 27, 233001

Bartelmann M., Schneider P., 2001, Phys.Rept., 340, 291

Bassett B. A., Kunz M., 2004, Phys. Rev., D69, 101305

Bradt H., 2004, Astronomy Methods: A Physical Approach to Astronomical Observations. Cambridge Planetary Science, Cambridge University Press

Civano F., et al., 2012, Astrophys. J., 752, 49

Ellis G. F. R., 1971, in Sachs R., ed., Proceedings of the International School of Physics Enrico Fermi. Course 47. pp 104-182

Ellis G. F. R., Bruni M., 1989, Phys. Rev., D40, 1804

Ellis G., Stoeger W., 1987, Class.Quant.Grav., 4, 1697

Etherington I. M. H., 1933, Phil. Mag., 15, 761

Frittelli S., 2003, Mon. Not. Roy. Astron. Soc., 340, 457

Frittelli S., Kling T. P., 2011, Mon. Not. Roy. Astron. Soc., 415, 3599

Frittelli S., Kling T. P., Newman E. T., 2000a, Phys. Rev. D, 63, 023006

Frittelli S., Kling T. P., Newman E. T., 2000b, Phys. Rev. D, 63, 023007

Gallo E., Moreschi O. M., 2011, Phys.Rev., D83, 083007

Gallo E., Moreschi O., 2012, Mod.Phys.Lett., A27, 1250044

Geroch R., Held A., Penrose R., 1973, J. Math. Phys., 14, 874

Gerosa D., Moore C. J., 2016, Phys. Rev. Lett., 117, 011101

Holanda R. F. L., Busti V. C., Alcaniz J. S., 2016, JCAP, 1602, 054

Kling T. P., Frittelli S., 2008, Astrophys. J., 675, 115

Kopeikin S. M., Schaefer G., 1999, Phys. Rev., D60, 124002 
Lambas D. G., Lares M., Ceccarelli L., Ruiz A. N., Paz D. J., Maldonado V. E., Luparello H. E., 2016, Mon. Not. Roy. Astron. Soc., 455, L99

Liao K., Li Z., Cao S., Biesiada M., Zheng X., Zhu Z.-H., 2016, Astrophys. J., 822, 74

Lokas E. L., Mamon G. A., 2003, Mon. Not. Roy. Astron. Soc., 343,401

Moreschi O. M., 1990, J. Math. Phys., 31, 1208

Perlick V., 2004, Living Rev. Rel., 7, 9

Perlmutter S., et al., 1999, Astrophys.J., 517, 565

Pyne T., Birkinshaw M., 1996, ApJ, 458, 46

Riess A. G., et al., 1998, Astron.J., 116, 1009

Riess A. G., et al., 2004, Astrophys.J., 607, 665

Sachs R., 1961, Proc. Roy. Soc. A, 264, 309

Schneider P., Ehlers J., Falco E., 1992, Gravitational lenses. Springer-Verlag

Schneider P., Kochanek C., Wambsganss J., 2006, Gravitational Lensing: Strong, Weak and Micro. Springer-Verlag

Seitz S., Schneider P., Ehlers J., 1994, Class.Quantum.Grav., 11, 2345

Stewart J. M., 1990, Class. Quant. Grav., 7, 1169

Stewart J. M., Walker M., 1974, Proc. Roy. Soc. Lond., A341, 49

Tichy W., Marronetti P., 2007, Phys. Rev.D, 76, 061502

Wald R., 1984, General Relativity. The Chicago University Press

Wambsganss J., 1998, Living Rev. Rel., 1

Wucknitz O., Sperhake U., 2004, Phys. Rev., D69, 063001

\section{APPENDIX A: GEOMETRY IN THE \\ COORDINATE SYSTEMS ADAPTED TO THE PAST LIGHT CONE}

In this appendix we present a list of the geometric quantities that we have employed for the description of the R-W spacetime in terms of the GHP formalism Geroch et al. (1973). We have taken the most relevant null tetrad for our purpose, namely those presented in subsection (3.1.2). For simplicity we present the expressions in terms of comoving coordinates.

Since Robertson-Walker geometries are conformally flat, its geometry is characterized only by its connection and the Ricci curvature since its Weyl tensor vanishes.

A1 The connection and the curvature for the null tetrad associated to the null function $u$

A1.1 Connection in terms of spin coefficients

$$
\begin{aligned}
\rho & =-\frac{A\left(t_{0}\right)}{A(t)}\left(\frac{H(t)}{c}-\frac{\sqrt{1-k f_{k}^{2}(\chi)}}{A(t) f_{k}(\chi)}\right), \\
\rho^{\prime} & =-\frac{A(t)}{A\left(t_{0}\right)}\left(\frac{H(t)}{c}+\frac{\sqrt{1-k f_{k}^{2}(\chi)}}{A(t) f_{k}(\chi)}\right), \\
\beta & =-i \frac{\sqrt{2}}{4} \frac{\zeta}{A(t) f_{k}(\chi)}, \\
\beta^{\prime} & =i \frac{\sqrt{2}}{4} \frac{\bar{\zeta}}{A(t) f_{k}(\chi)} \\
\epsilon^{\prime} & =\frac{A(t)}{2 A\left(t_{0}\right)} \frac{H(t)}{c} .
\end{aligned}
$$

\section{A1.2 Ricci curvature scalar}

$$
\begin{aligned}
\Phi_{00} & =\frac{A^{2}\left(t_{0}\right)}{c^{2} A^{2}(t)}\left(\frac{k c^{2}}{A^{2}(t)}+H^{2}(t)+q(t) H^{2}(t)\right), \\
\Phi_{11} & =\frac{1}{4} \frac{A^{2}(t)}{A^{2}\left(t_{0}\right)} \Phi_{00}, \\
\Phi_{22} & =\frac{1}{4} \frac{A^{4}(t)}{A^{4}\left(t_{0}\right)} \Phi_{00}=\frac{A^{2}(t)}{A^{2}\left(t_{0}\right)} \Phi_{11}, \\
\Lambda_{\mathrm{GHP}} & =\frac{1}{4 c^{2}}\left(\frac{k c^{2}}{A^{2}(t)}+H^{2}(t)-q(t) H^{2}(t)\right) .
\end{aligned}
$$

Some of these equations are found in Moreschi (1990)

This paper has been typeset from a $\mathrm{TE}_{\mathrm{E}} \mathrm{X} / \mathrm{AT} \mathrm{T} \mathrm{X}$ file prepared by the author. 